\title{
Building A blockchain-based decentralized digital asset management system for commercial aircraft leasing
}

\author{
Paul Kuhle ${ }^{\mathrm{a}, *}$, David Arroyo ${ }^{\mathrm{b}}$, Eric Schuster ${ }^{\mathrm{c}}$ \\ a Universidad Autónoma de Madrid, Facultad de Ciencias Económicas y Empresariales, Dept. of Finance, Calle Adam Smith 2, 28049 Madrid, Spain \\ ${ }^{\mathrm{b}}$ Instituto de Tecnologías Físicas y de la Información (ITEFI), Consejo Superior de Investigaciones Cientificas (CSIC), 28006 Madrid, Spain \\ ' Technische Universität Berlin, Dept. of Aeronautics and Astronautics, Chair of Flight Guidance and Air Transport, Marchstr. 12, 10587 Berlin, Germany
}

\section{A R T I C L E I N F O}

\section{Article history:}

Received 25 June 2020

Received in revised form

10 November 2020

Accepted 29 December 2020

Available online 11 January 2021

\section{Keywords:}

Blockchain

Hyperledger

Aircraft leasing

Smart contracts

Privacy

Internet of Things

\begin{abstract}
A B S T R A C T
The blockchain technology has the potential to drastically change the way record-keeping and asset management is done. While more and more aerospace companies are starting to adopt the new technology, commercial aircraft leasing can still be considered uncharted territory for blockchain applications. The present paper analyzes how the sector stands to benefit from such a solution and proposes a suitable blockchain application design, focusing both on the regulatory and business needs as well as the implementation from a technological perspective. Our proof-of-concept demonstrates that asset management in commercial aircraft leasing can realistically be handled by a blockchain solution such as the one proposed in this paper.
\end{abstract}

(c) 2021 Elsevier B.V. All rights reserved.

\section{Introduction}

Commercial aircraft are often leased instead of purchased directly to either satisfy short-term demand spikes or as part of a long-term fleet planning strategy, where aircraft leasing is used as a tool to increase flexibility and free up capital (Kehoe and Hallahan, 2017). The importance of leasing contracts has been growing rapidly: By the end of the decade, roughly half of all commercial aircraft worldwide will be operated under a leasing agreement (IATA, 2017, p. 1).

In an environment where multiple parties operate with conflicting interests, trust cannot be taken for granted. It is therefore more than advisable to elude the dependency on only one or a reduced set of parties. Instead, the deployment of means to articulate distributed trust management should be promoted. Moreover, there is an urge, and even a normative and legal framework targeted to promote transparency and governance in commercial aircraft operations.

The blockchain technology has the potential to drastically improve the efficiency and security of supply chains in general

\footnotetext{
* Corresponding author.

E-mail addresses: paul.kuhle@inv.uam.es (P. Kuhle), david.arroyo@csic.es (D. Arroyo), eric.schuster@tu-berlin.de (E. Schuster).
}

(Forum W.E., 2019), and particularly in the case of commercial aircraft leasing. Our proposal for a decentralized solution builds upon trust-based approaches like the Global Aircraft Trading System (GATS) (Aviation Working Group, 2020). Specifically, we show how the blockchain technology can be leveraged to properly align the business model and the legal and normative requirements. This solution aims to mitigate the shortcomings and challenges of today's asset management solutions. These include inefficient processes, auditing issues and reduced asset management capabilities due to a lack of real-time information.

The objective of this paper is not to develop a software product or a blockchain application, but rather to propose a blueprint for a decentralized ecosystem for commercial aircraft leasing and to show how it integrates into the broader IT landscape in civil aviation.

In Section 2, we take a look at the current challenges that the industry is facing and how the blockchain technology can match the industry's business needs and regulatory requirements. We then present our proposal in Section 3.

\section{Theoretical background}

The following sections will give a brief overview of the key terms and ideas as well as the state of the art processes used in commercial 


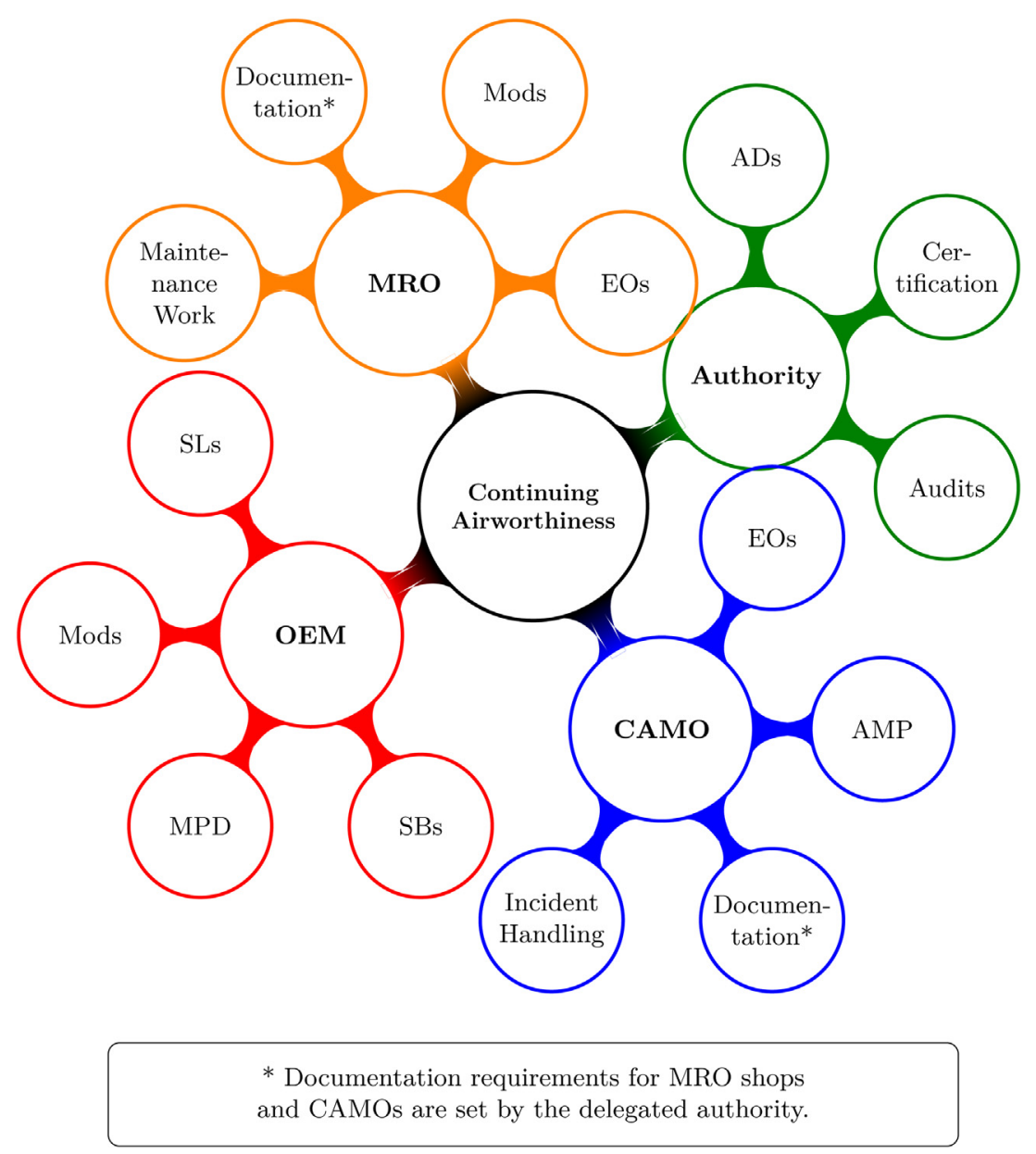

Fig. 1. Continuing airworthiness stakeholders.

aircraft leasing. First of all, it is discussed the regulatory environment (Section 2.1) and the individual stakeholders' responsibilities and needs in commercial aircraft leasing (Section 2.2). After that, the main challenges in commercial aircraft leasing are highlighted in Section 2.3 in order to be tackled by means of blockchain in Section 2.4 .

\subsection{Commercial aircraft leasing and its regulatory environment}

Aircraft leasing only affects the operational phase of an aircraft's ${ }^{1}$ lifecycle. In this phase, the operator's utilization of an aircraft is subject to Continuing Airworthiness (CA) regulations to ensure minimum operability. Legally, the operator is not allowed to assume responsibility for the technical condition of their own fleet. Thus, a multi-facetted stakeholder setup is created around the operator (see Fig. 1):

- the authority/regulator (and the delegated national authorities, e.g. the German Luftfahrtbundesamt),

- the Original Equipment Manufacturers (OEMs),

- the Continuing Airworthiness Management Organization (CAMO) set up or contracted by the operator of the aircraft and

- any Maintenance, Repair and Operations (MRO) shop that performs scheduled and unscheduled maintenance (MX) work on the aircraft, its components or its engines.

\footnotetext{
1 A complex motor-powered aircraft as defined in (EC, 2008, Art. 3j).
}

In Europe, CAMOs and MRO shops are governed by European law and the European Aviation Safety Agency (EASA) (EU, 2014). The certification process is handled by the delegated authority which also oversees and audits all involved parties. Safety-critical findings are communicated to the authorities and the OEMs, who can issue the following publications:

- Airworthiness Directives (ADs) are published by authorities and contain mandatory instructions that need to be followed by the CAMO.

- Service Bulletins (SBs) are issued by the OEM and contain modifications and design improvements. SBs are not mandatory unless they are referenced in an AD. Other changes are often installed as modifications (Mods).

- Service Letters (SLs) and Servive Information Letters (SILs) are used to share non-critical information and documentation between the involved parties.

Consequently, different aircraft of the same type can vary significantly in equipment and component configuration. It is crucial to keep track of an aircraft's build stand, i.e. which ADs, SBs and other modifications have been installed. ADs may or may not affect an aircraft depending on its build stand, serial number or utilization.

Additionally, the CAMO is responsible for maintaining the aircraft in airworthy condition by following an Approved Maintenance Plan (AMP) on an organizational level and handling unscheduled incidents. AMPs can follow the Maintenance Planning Document (MPD) provided by the OEM. Any changes to the MPD must be approved by the authority, so that the CAMO's work complies with a 


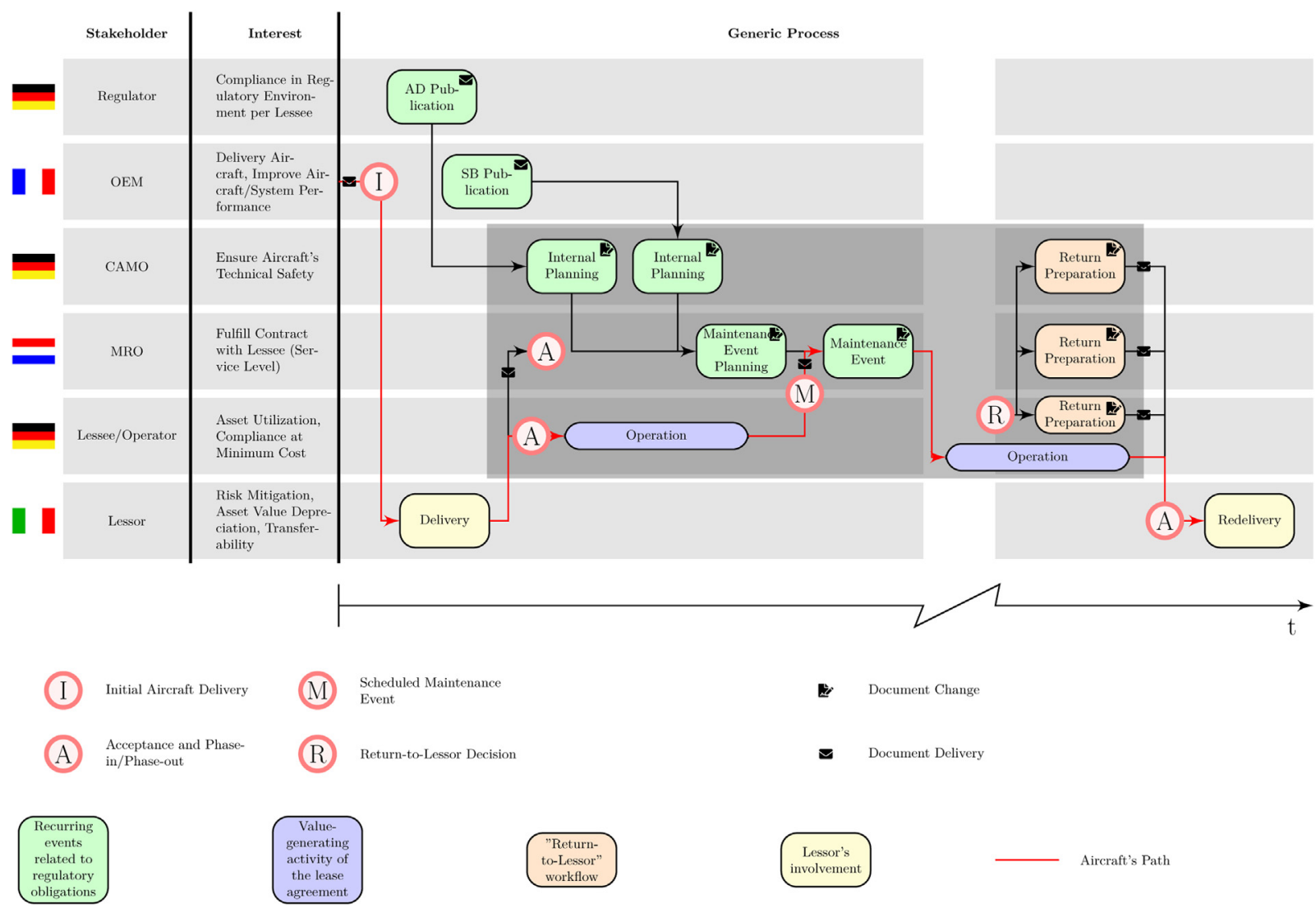

Fig. 2. Diverging interests of the stakeholders involved in commercial aircraft leasing across a simplified, generic process.

list of applicable MX processes, tasks and their intervals. Applicable tasks are carried out via Engineering Orders (EOs).

The MRO organization performs the actual MX work on the aircraft. The MX plan contains the scheduled Job Cards (JCs) and unscheduled Job Orders (JOs), and consists of line and base MX events, varying from $20 \mathrm{~h}$ to several months. MX events can cause diverse changes in aircraft composition depending on the amount and ratio of JCs and JOs solved per event (Transport Studies Group, 2008, Tables 1 and 2). After the MX work is completed, the aircraft is returned to the operator and put back into service.

The stakeholders can be based in different geographic locations and legal jurisdictions. This introduces more complexities with regards to non-standardized legislation, security concerns and privacy policies of the stakeholders' countries of origin. The exact requirements also depend on the lease type (dry or wet lease as defined in (EU, 2018, Art. 2, 24f.)). This paper focuses on a standard dry-lease agreement, as the stakeholder relationships of a wet-lease agreement are more trivial in comparison.

\subsection{Business needs in commercial aircraft leasing and current state-of-the-art processes}

The generic aircraft leasing cycle comprises a complex of stakeholders and processes which not always share interests and goals (see Fig. 2). After ordering an aircraft from an OEM, the lessor will take delivery of the aircraft and its documentation if all acceptance criteria are met. At this point, the lessor has complete knowledge of the technical configuration of the aircraft. Subsequently, the aircraft can be introduced into a lessee's fleet, at which point the asset leaves the lessor's legal jurisdiction.

Before aircraft (re)delivery, detailed inspections must be performed to guarantee compliance with financial, legal and with safety conditions (aircraft's physical condition, MX status and documentation).

During the lease, the lessee uses lessor-approved MRO shops to follow the AMP as agreed by both the regulator and the lessor. On the basis of the leasing contract, the lessors must document the aircraft's life to enable auditing of authority, responsibility and accountabilty. Internally, the lessee, CAMO and MRO organizations often use commercial MRO software like AMOS to share information about business processes like logistics, operational planning and controlling. This workflow (highlighted in black in Fig. 2) is opaque from the regulator's, lessor's and OEM's point of view. To gain insight into this black box, they can perform audits whose efficacy and efficiency depend on the organization's capability to produce the required records. Although extensions like FlyDocs endow MRO software to fulfill this goal, current implementations mainly rely on the digital replication of paper-based documentation.

A typical leasing contract goes beyond Fig. 2 and covers payments, risk mitigation and operational restrictions. These elements are usually negotiated freely between the lessor and the lessee and often involve additional stakeholders like insurance brokers, consulting firms, payment processors and other financial institutions. From an asset and lifecycle management point of view (Hanley, 2011, pp. 32-37), a leasing contract consists of four key elements: Delivery and Redelivery, Operations, Payments and Risk (see Fig. 3).

The lessee's monthly lease rate consists of a base rate and an additional rent. The additional rent covers the lessee's contributions to future MX events derived from the lessee's utilization of the aircraft and to be carried out after the lease expires (e.g. structural work is often carried out in intervals of 10 years or more, which greatly exceeds the average lease term). 


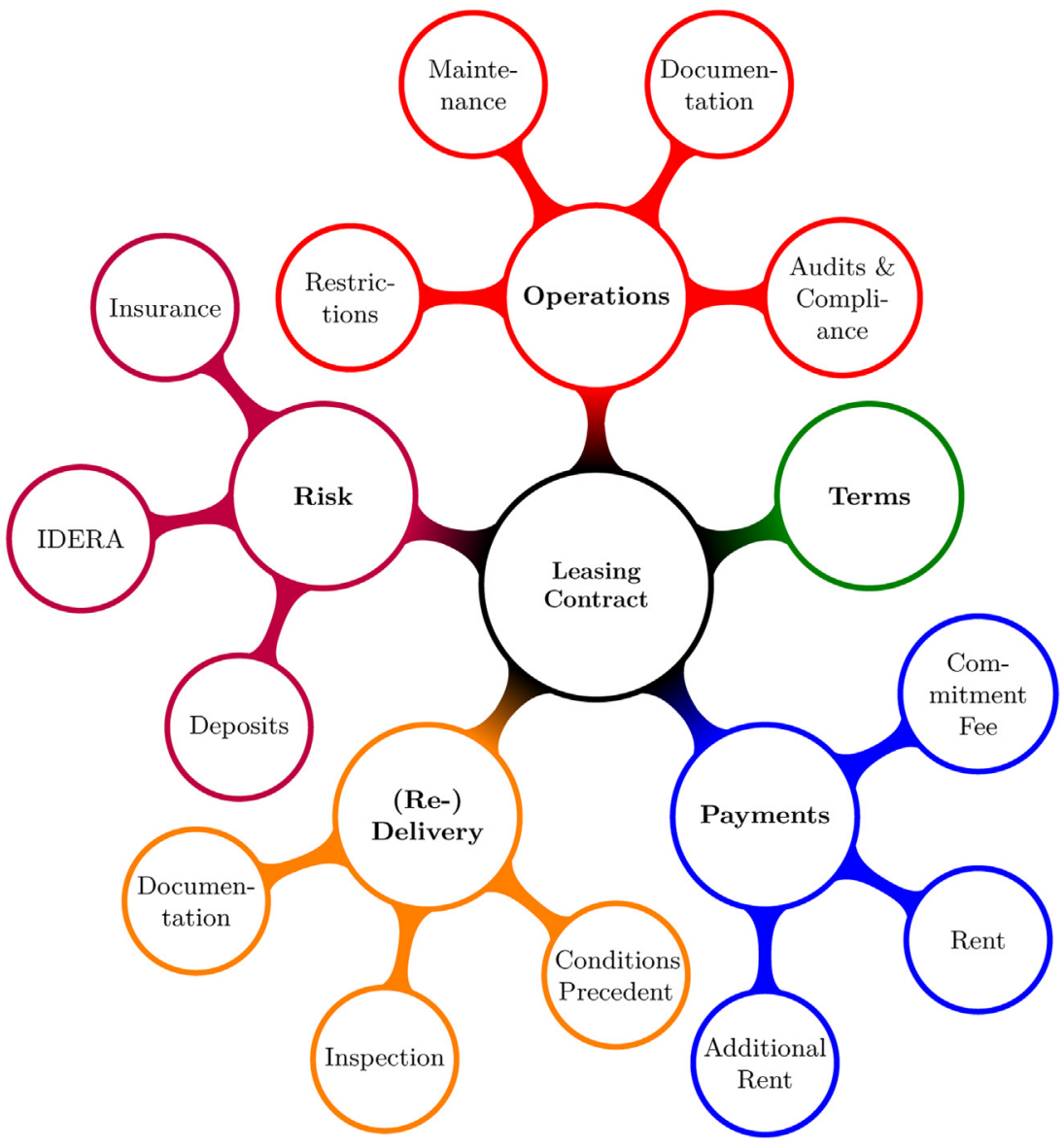

Fig. 3. Leasing contract components.

The three main tools to mitigate risk in a lease agreement are security deposits, insurance and the Irrevocable De-Registration and Export Request Authorization (IDERA), which provides an international legal regime to govern security interests in aircraft (ICAO, 2001). It allows creditors to repossess an aircraft in case the lessee defaults on his payments. Additional operational restrictions may be imposed by the lessor during the lease (e.g. the lessee is not allowed to change the habitual base of the aircraft to another country or to fly the aircraft into active warzones or states sanctionted by the United Nations Security Council).

While there have been efforts to standardize at least parts of aircraft lease agreements, most lessors have their own proprietary leasing contract templates (IATA, 2012). This paper will focus on a generic leasing contract and a generic timeline based on publicly available information.

\subsection{Current challenges}

The current approach to document-sharing carries a certain risk of fraud, document loss and auditing issues, even in developed countries (FAA, 2019). Signatures and stamps on physical documents and their digital replicas, if they exist, add little value to an external auditor. Company-internal identity management systems are not linked to government-issued identifications or external certifications that the auditor may require. Tracing the internal chain of responsibility in the organization seems impossible under today's bottom-up MX approach as company-internal processes cannot be monitored by outside stakeholders (i.e. regulators and lessors). Indeed, the current system is missing real-time information about the aircraft's operational and CA activity, which hampers the regulator's ability to ensure compliance with the MX obligations outlined in Section 2.1. As of today, the regulator has to rely on costly and, given the mentioned issues, potentially unreliable audits. This problem also affects downstream stakeholders like MRO organizations and suppliers that are subject to similar audits.

Besides operational safety, as part of the asset management strategy, the lessor must deliver the aircraft to the next lessee in airworthy condition. Due to the current lack of automation, the lessor first gets a chance to verify maintenance documents at lease expiry. Consequently, at this stage the exhaustive inspection of the aircraft and its documentation leads to a significant workload. According to the timeline proposed by IATA (IATA, 2017, pp. 58-61), the whole redelivery process takes more than a year for a long-term lease. Operational restrictions and maintenance reserves can often only be checked, enforced and collected when the aircraft is physically redelivered, even with IDERA.

Another concern arises from the selection of adequate Information Technology (IT) architectures for the distributed and sensitive nature of the aircraft leasing ecosystem. Current approaches like the GATS (Aviation Working Group, 2020) adopt a centralized management scheme, which could erode stakeholders trust in the IT system. Indeed, the stakeholders of our ecosystem often need to gather information from other stakeholders whose interests can be diametrically opposed.

A centralized global solution would likely have insufficient international backing. In fact, countries like Russia and Iran have been subject to sanctions affecting their civil aviation industry (Nadimi, 2019) and their trust in centralized global institutions like the United Nations and their suborganizations like the International Civil Aviation Organization (ICAO) is limited (Radin and 
Reach, 2017, pp. 39). This solution could therefore only capture a portion of the market and will always be limited by the trust that other stakeholders (and even individual employees as part of a criminal liability in MX fraud or irregularities) have in the solution's operator.

\subsection{Technological capabilities of blockchains in the context of commercial aircraft leasing}

The functionality of the blockchain technology can help to tackle operational and tactical aspects in aircraft leasing, and also to bolster new strategical schemes. Blockchains are append-only lists of blocks in which validated transactions are stored chronologically (Narayanan and Clark, 2017). Each new block contains a certain number of transactions. When a block reaches that target number of transactions, it is added to that list, thereby generating a ledger of all conducted transactions. This ledger is not stored in a central database; instead it is stored on every peer in the network. Moreover, the information written in a blockchain cannot be easily unilaterally modified, which means that the information persisted in a blockchain can be regarded as immutable (unless enough peers collude).

Beyond the chain supply management in the airport industry ( $\mathrm{Di}$ Vaio and Varriale, 2020), asset traceability and data provenance are fundamental in the management of aircraft leasing. The tamperproof nature of the blockchain and the chronological recording in the blocks are very useful to foster audit and accountability procedures (Forum W.E., 2019). The capability of creating a trust layer that is independent from the rest of the communication protocols stack is of major relevance, since it enables disintermediation and reduces the dependency on third parties along the lifecycle of the leasing process. As it has been widely developed in financial networks (Yermack, 2017), blockchains endorse collaborative decision making and taking processes, which reduces the dependency on single entities or organizations. This is also of high relevance in the field of cybersecurity, where collaboration between actors must be conducted according to convenient data minimization schemes (Rubio et al., 2020).

Many airlines are already using blockchains, but only for specific tasks. For example, Russian-based S7 Airlines is handling more than $\$ 1$ million in monthly B2B payments, e.g. for aircraft refueling, via a blockchain platform (S7 Airlines, 2019). Blockchains can help to automate other business processes by means of smart contracts (IATA, 2018). Smart contracts are coded agreements that are stored in the immutable blockchain to be automatically executed once a set of conditions is satisfied. This capability enables machine-tomachine interaction, which is a requirement in the deployment of advanced solutions for the Internet of Things (IoT) (Lao et al., 2020). As another application context in the Industry 4.0, the aircraft leasing industry can benefit from the tokenization of assets and their management by smart contracts.

Decentralized approaches that encourage the efficient and safe sharing of information and documentation have the potential to drastically improve lifecycle and asset management in commercial aviation because they can provide trust between parties that otherwise have little reason to trust each other. Identity management is crucial to attain these goals, and it demands the articulation of robust and efficient access and authorization models. In this regard, we have to take into account that blockchains can either be public, private or a consortium (see Table 2). A public blockchain allows anyone, barring hardware restrictions, to set up a node on his computer and read data from the blockchain. Private chains use whitelist filters to make the data available only to selected individuals. In a private blockchain, these peers are typically controlled by one single company, making this type of blockchain less suited for truly decentralized applications. Some application contexts call for an adequate balance between decentralization and accountability. In this regard, a consortium blockchain (also known as hybrid blockchain) is a viable solution. Consortium blockchains are built around the idea of allowing access only to a few peers from different companies.

Finally, we have to bear in mind that blockchain is a means to persist information and to ease its sharing after being stored. The source of the information is external and, consequently, the interface using during the recording stage is not protected by the tamper-proof characteristics of the blockchain. Data curation in general (Heiss et al., 2019), and particularly in the case of legal compliance and liability (Arribas et al., 2020) demands the inclusion of off-chain actors and/or agents. Therefore, the reliability and trustworthiness of these actors should be further analyzed (Yang et al., 2020).

\section{The proposal}

The requirements for our concept are derived from the regulatory environment, the business needs and the challenges that the aircraft leasing industry is currently facing.

The functional requirements can be seen in Table 1 . The non-functional requirements fall into the categories Compliance (Table 3), Performance (Table 4), Business Needs (Table 5) and Usability (Table 6).

In this section, we propose a blockchain architecture that implements these requirements.

\subsection{Blockchain platform}

This project calls for a consortium blockchain, given that commercial aircraft leasing is a multi-organizational application. The blockchain frameworks that could potentially be used to develop this type of application are Ethereum and Hyperledger Fabric or Sawtooth. For this application, Hyperledger Sawtooth can be recommended, as it can fulfill the non-functional requirements better than Hyperledger Fabric and Ethereum:

- Hyperledger is backed and being contributed to by industry leaders like Airbus, Intel, IBM and Consensys (Hyperledger, 2020), making Hyperledger frameworks more maintainable in the long term (E-4). The involvement of smaller IT companies and consulting firms like Accenture means that Hyperledger has already developed an enterprise ecosystem (E-3).

- A blockchain built with Hyperledger Sawtooth can incorporate customized nodes that can be written in a number of different programming languages. Sawtooth does not require a specific deployment setup or data access architecture. This reduces vendor lock-in and increases overall flexibility (E-2).

- The Proof of Elapsed Time (PoET) consensus protocol available in Sawtooth makes use of a much more efficient algorithm than Proof of Work, which is used in Ethereum. Since the algorithm is a major driving force behind the blockchain's overall power consumption (Li et al., 2019), the PoET protocol offers an advantage with regards to $\mathbf{D - 2}$.

- The PoET protocol is Byzantine fault tolerant (BFT), i.e. it can reach consensus even when the system has malicious or faulty nodes. This is not offered by Hyperledger Fabric's Kafka protocol. PoET is also more scalable than Kafka (The Hyperledger Project, 2017) (C-1).

- Sawtooth has built-in access control and user management, but allows the use of neutral data sources and off-chain storage (D-1).

- Given Hyperledger Sawtooth's compatibility with Ethereum smart contracts, crypto-payments and other public blockchain services can be integrated easily (E-1). 
Table 1

Functional requirements.

\begin{tabular}{|c|c|c|}
\hline $\mathrm{Nr}$ & Title & Description \\
\hline A-1 & Tokenization & The physical asset (the aircraft) will be tokenized, i.e. issued a digital counterpart. \\
\hline A-2 & User management & $\begin{array}{l}\text { A user management system needs to be implemented to handle access rights and } \\
\text { stakeholder interactions. }\end{array}$ \\
\hline A-3 & Process automation & $\begin{array}{l}\text { The processes and interactions shown in Figs. } 2 \text { and } 3 \text { should be implemented as smart } \\
\text { contracts. }\end{array}$ \\
\hline A-4 & Interfaces & $\begin{array}{l}\text { The system needs to provide real-time access to relevant data. Regulators should be } \\
\text { given access to MX records, while lessors should also be able to audit flight logs, } \\
\text { equipment lists, the build stand, etc. Interfaces should be provided to the respective } \\
\text { stakeholders. }\end{array}$ \\
\hline
\end{tabular}

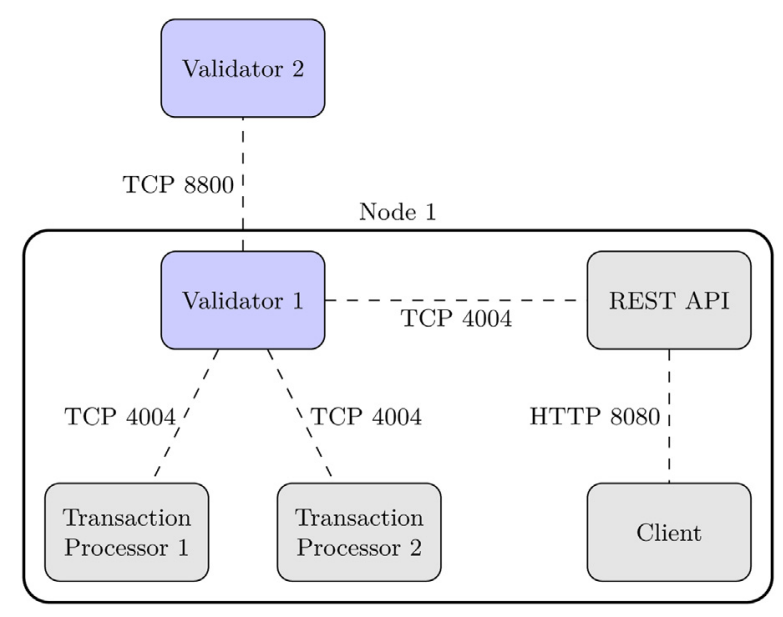

Fig. 4. Overview of Hyperledger Sawtooth Components. Adapted from (Intel Corporation, 2019).

Fig. 4 shows a generic overview of the Hyperledger Sawtooth components. Each node in the network contains a validator, one or more transaction processors (TP) and a REST API.

The validator contains the blockchain data and communicates with the other validators in the network to approve the transactions sent by the TPs. The TPs contain the business logic of the blockchain, i.e. functions to interact with blockchain data as well as the appropriate data models to encode and decode data that is stored on the blockchain.

In order to communicate with external applications, a Hyperledger Sawtooth node needs to have a REST API. The REST API handles transaction requests and event broadcasting, i.e. it sends out a signal to inform subscribers about state changes and other important events. This architecture makes it possible to tailor clients to the customer's needs.

\subsection{Proposed design}

\subsubsection{Architecture}

The architecture of the proposed solution can be seen in Fig. 5. Hyperledger Sawtooth already implements the protocol layer, including the decentralized data storage layer, the consensus mechanism and user management system.

The application layer contains the business logic as described in Section 3.1. This TP contains data models for the aircraft, the leasing contract and the AMP (A-1). Stakeholders can interact with these data models according to their individual interests and responsibilities outlined in Section 2 using their respective transaction families (A-3) as shown in Fig. 6. Transaction families may need to query external data sources as presented in Fig. 5. Custom APIs and clients are added as an abstraction layer (A-4).

\subsubsection{User management}

One of the key features is user management (A-2), which is commonly implemented as Authentication, Authorization and Accountability (AAA). This application uses an asymmetric cryptography system to ensure robust user authentication, creating a public and private key pair for every stakeholder that has to interact with the blockchain. Once the user is authenticated, they may need authorization to perform certain tasks. Hyperledger Sawtooth provides three different methods to handle these permissions:

- Allowed transaction families. The network operator can specify which types of transactions can be submitted, making the network ignore unrecognized TPs. This application only recognizes the internal Hyperledger Sawtooth transaction families and the custom TP (aero) that was developed in this work.

- Validator permissions. This functionality can be used to specify the nodes that are able to connect to the network.

- Transactor permissions, which are used to define who can submit transactions to a validator. Permissions can be set offchain (locally) or on-chain (network-wide). A transaction is only allowed if both the off-chain and on-chain settings agree that it should be accepted. For instance, an MRO shop may be given restricted transaction rights in the on-chain settings. The MRO shop could use off-chain settings to further restrict transaction rights within their own organization, e.g. to give engineers and mechanics different permissions.

For the sake of network security, stakeholders were only given transaction signer rights for the aero transaction family. Moreover, Hyperledger Sawtooth fosters accountability. First, the underlying blockchain stores all transaction receipts in an immutable and transparent way. Second, any single transaction is digitally signed and therefore it is possible to trace back actions to individual identities.

\subsubsection{Data models}

While all data is stored on-chain within the validator, data models are needed to make sense of it. The validator only stores serialized data, i.e. in a series of bits that is suitable for transmission and storage. Google's Protocol Buffer format is used as the serialization format. Protocol Buffers are language-neutral, platform-neutral, fast and lightweight (Google LLC, 2019). They also offer deterministic serialization, which is important given that the validator enforces deterministic behavior in the TP and data models.

In this application, the public keys used for user authentication are also used for address handling. Hyperledger Sawtooth uses 35bit addresses to identify the position of each portion of data within the blockchain's data storage system (see Fig. 7). For most stakeholders, their public key can be used to generate a unique address. Other entities like aircraft do not have a public key, as they do not represent a stakeholder. These addresses need to be generated 
Data Models
Transaction Families
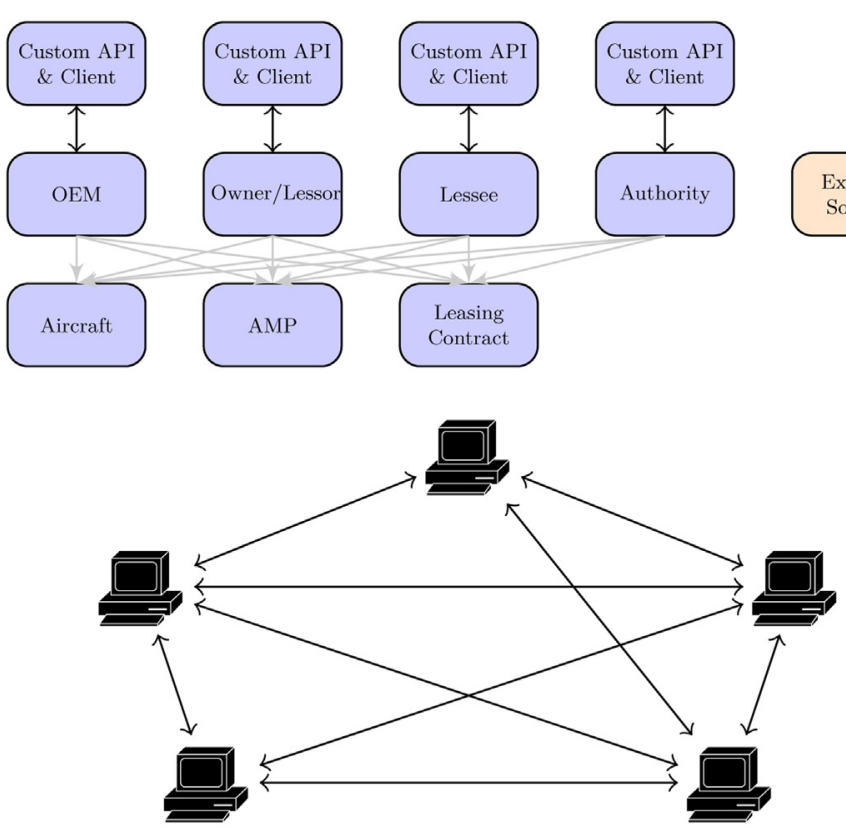

Network:

Consensus Protocol

User Management

Data Storage
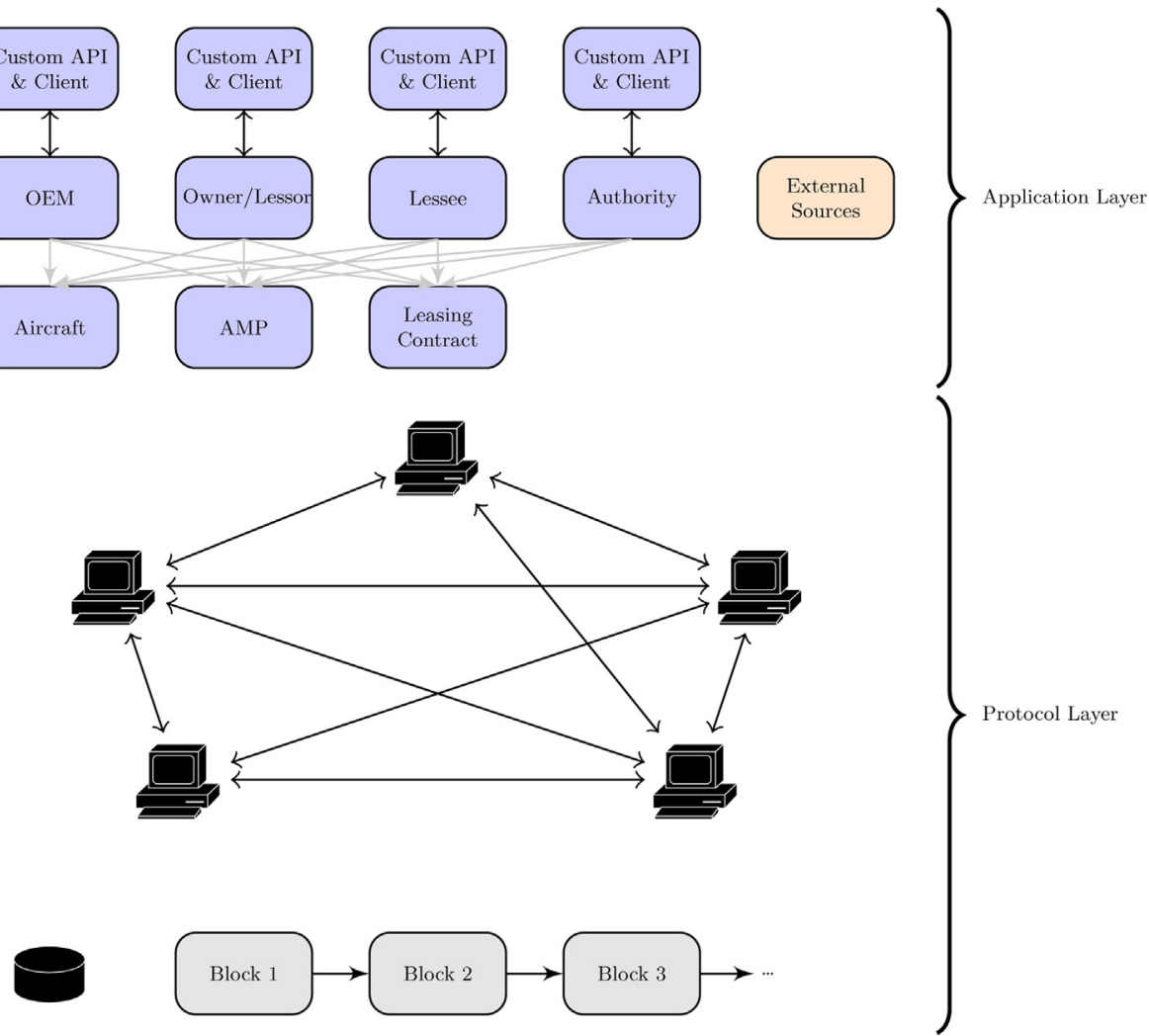

Fig. 5. Architecture overview.

using model data, e.g. by hashing the aircraft type and Manufacturer Serial Number (MSN).

The stakeholders have similar data models that store their public key, name and country. These models can be inherited from and expanded as needed to store additional information. As an example, the data model for an aircraft owner/lessor can be seen in Code Block 1.1.

The aircraft data model stores the stakeholder history (OEMs, owners, operators, authorities), the flight, equipment, MRO event, $\mathrm{AD}, \mathrm{SB}$, location, hours, cycles and audit histories as well as some basic data like its own address, MSN and engine type. It also stores a list of associated AMPs and leasing contracts. The model can be expanded as well, but the provided generic implementation in Code Block 1.2 is sufficient to fulfill B-3.

The AMP data model stores MX tasks and their applicability, thresholds and associated maintenance events. The implementation shown in Code Block 1.3 is fairly generic and can be used with any AMP.

As discussed in Section 2.2, there is no generic or industrystandard leasing contract. It would not be sensible to force a standardized contract scheme onto the lessor and lessee, who are free to negotiate their own terms, restrictions and conditions. Since the business relationship between the lessor and the lessee does not concern any other stakeholders, they need to be able to set up their own transaction families and data models. Sample contracts like the one used in this proof-of-concept (Code Block 1.4) or individual modules like geographic restrictions can be provided.

\subsubsection{Data storage}

Because blockchains are not meant to store large amounts of data, the data models are not used to store large files like PDF documents that are often necessary to properly document contracts, MX events and audits. Instead, the blockchain stores a link (or anchor) to the file. This anchor is usually contructed using a hash function (e.g. SHA-512), and it can be used to ensure that the file has not been modified after the initial upload.

For simplicity's sake, this paper assumes that all files are stored off-chain in a location chosen by the data owner (e.g. their own servers or a cloud solution like AWS). This location would need to be compatible with the Simple Storage Service (S3) protocol. A dedicated transaction family is then used to generate pre-signed URLs to protected S3 files. The key used to generate this URL would only be stored on the data owner's node. The blockchain would simply act as a consensus mechanism to determine access rights. This process is shown in Fig. 8.

This solution satisfies B-1, B-2 and D-1. However, it is not a truly decentralized solution. While the blockchain determines access rights, the data owner could always overwrite this in his node's settings or in his storage server settings.

A better approach would be to employ a distributed file storage solution (Huang et al., 2020).

\subsubsection{Transaction processor}

As shown in Fig. 9, the TP consists of a transaction handler and a state handler. The transaction handler is responsible for deserializing the incoming transaction payload, verifying the received data and generating the container data that will be recorded on the blockchain. State changes are handled by the state handler, which serializes the container data according to the specifications in the data models and submits the state change.

The most complex and computationally expensive parts of the process are the verification of transaction data and the generation of new container data. For instance, when a new flight is added to the aircraft's flight log, the transaction handler has to verify the items shown in Fig. 10.

Some of these items require further input from neutral data sources (orange boxes) or other blockchain data models (green boxes). The transaction handler can invoke other TP functions, e.g. 


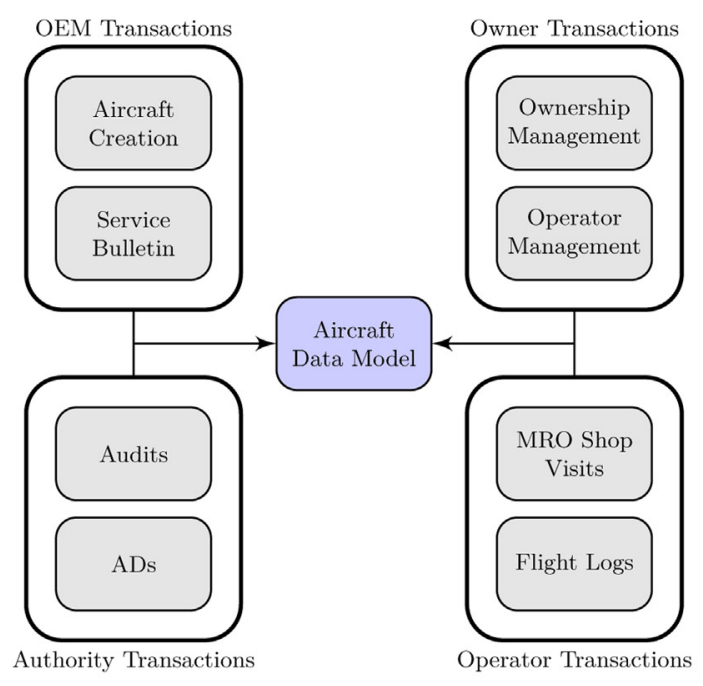

(a) Aircraft

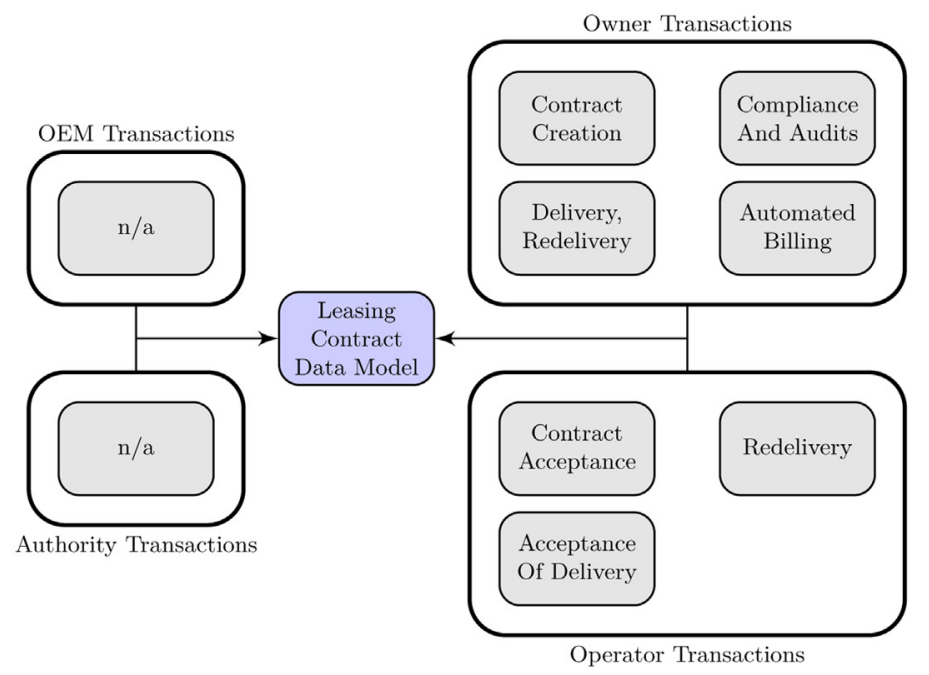

(c) Leasing Contract

Fig. 6. Data model interactions.

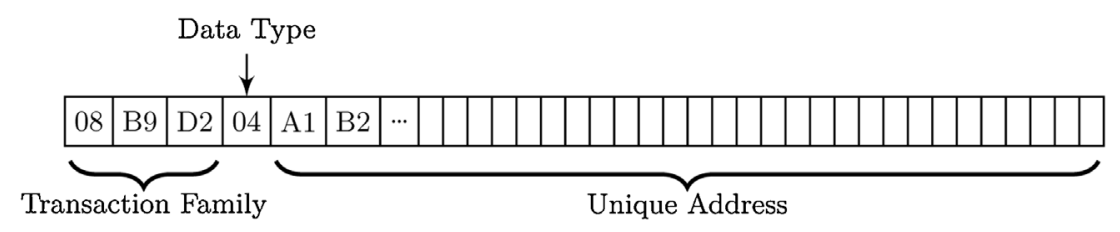

Fig. 7. Address handling in Hyperledger Sawtooth.

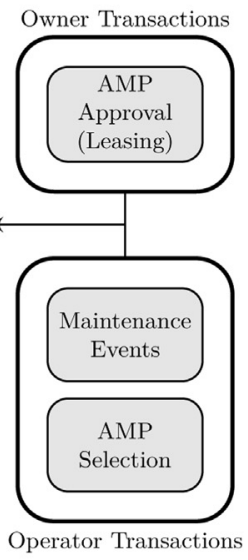




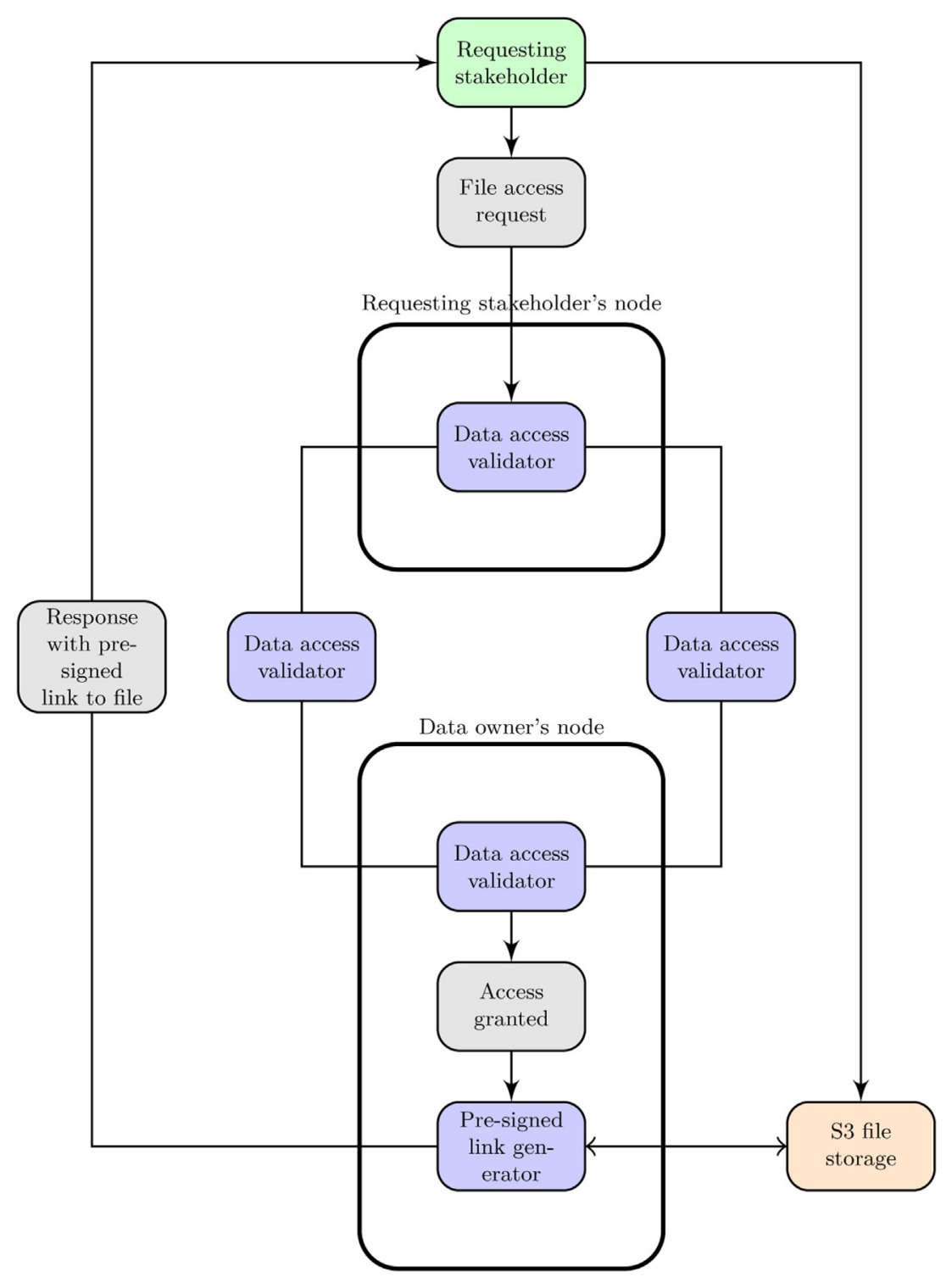

Fig. 8. Off-chain data storage.

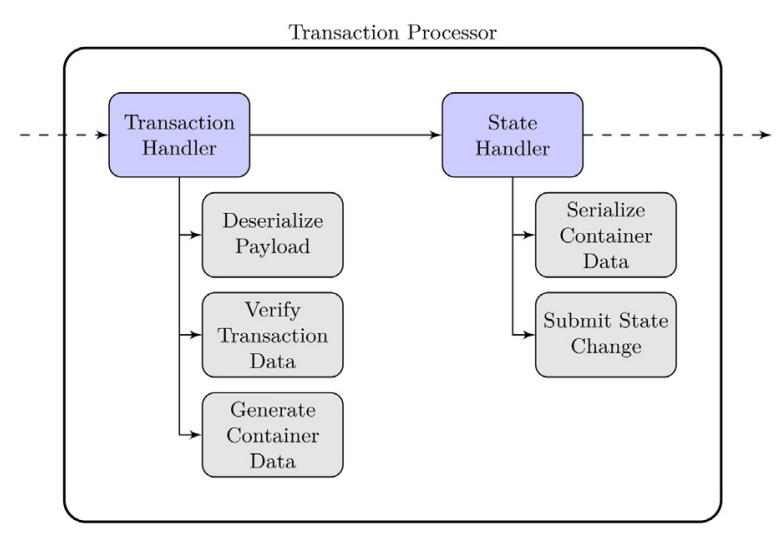

Fig. 9. Transaction processor layout in Hyperledger Sawtooth.

to $\log$ a violation of a leasing contract's operational restrictions. It is also possible to write to multiple fields and data models in the state handler. For example, when a new flight is added, the state handler adds the flight to the aircraft's flight log, but also updates the flight cycle entries.
The verification of transaction data can impose a significant load on the node, especially when large databases and/or external data sources are involved. It becomes apparent that a well-designed TP can have a big impact on overall node performance. It should be noted that:

- Many utility functions will likely be called very frequently.

- The TP handler functions will be executed repeatedly and on multiple nodes for every submitted transaction to ensure that the function is indeed deterministic.

\subsubsection{REST API \& client}

The REST API provides an interface to communicate with the blockchain. It serializes the transaction payload before forwarding it to the node. The protocol buffers used to serialize the flight insertion payload are shown as an example in Code Block 1.5. The request also includes inputs and outputs fields that define the addresses that the TP is allowed to read from and write to.

It is possible to include generic addresses in those fields. This can be useful for transactions where the address cannot be fetched or calculated by the API, but it can lead to drastic performance decreases if the data model has a high transaction throughput. This 


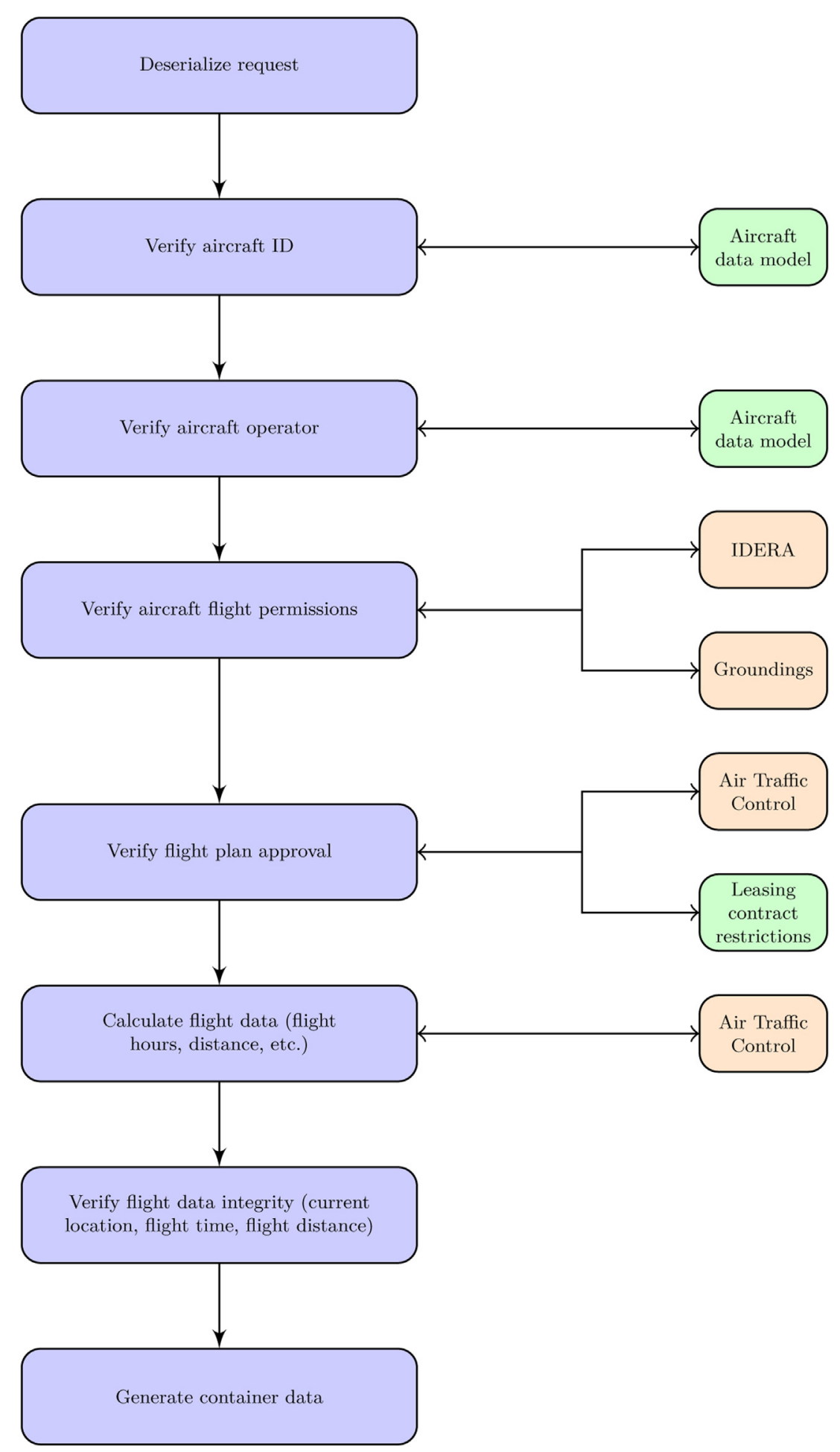

Fig. 10. TP flowchart for flight data insertion. (For interpretation of the references to color in the text, the reader is referred to the web version of this article.)

is due to the fact that the TP cannot schedule parallel transactions for any addresses that are currently in use.

The client that was developed for this paper is a generic implementation for human end users. It operates independently from the node and can be set up according to the customer's needs. An overview of the different components of this client can be seen in Fig. 11. A subscriber service listens to the REST API and writes new state changes to a PostgreSQL database. This design allows for quick access times to blockchain data, since the frontend app never needs to query the blockchain's REST API directly. The subscriber can make a catch-up request to the REST API in case it is missing blocks, e.g. when a new client needs to be synchronized.

The synchronized data models can be customized and enriched with data from other sources. A sample dashboard page is shown in Fig. 12. It lists the aircraft's stakeholders and their blockchain addresses together with a visual representation of the aircraft's flight cycle/flight hours history and information about its last flight.

The demo application also includes the following detail pages: 


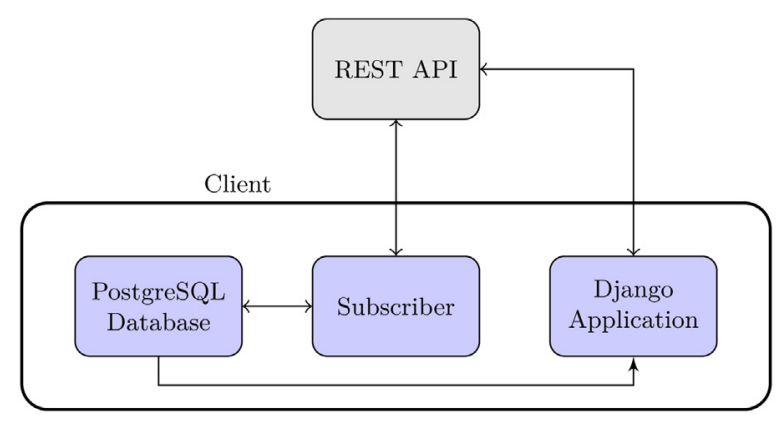

Fig. 11. Client architecture.

- The aircraft's flight log (Fig. 13).

- An AMP page with a list of tasks, events, MRO shop visits and compliance entries (Fig. 14).

- The aircraft's equipment history (Fig. 15).

- A leasing contract page with information about the delivery and redelivery, payments (Fig. 16) and compliance (Fig. 17).

\subsection{Discussion}

In order to validate the functional and non-functional requirements, we implemented the proposal presented in Section 3 in Python as a proof-of-concept and simulated several aircraft leases.

In order to ensure the accuracy of the simulation, the workload was generated using actual flight data and a complete MX task list taken from a real MPD. The flight data includes all commercial flights operated in 2018 by Finnair's and Royal Jordanian's Airbus A320 family fleet. These airlines were chosen because their combined fleet size of 50 aircraft is large enough to conduct scalability tests and because the two airlines operate in different jurisdic- tions. This is required for certain functional tests like transferring an aircraft to an operator that is regulated by a different authority.

The simulation has shown that the proposed design fulfils the functional requirements defined in Table 1.

One major downside of Hyperledger Sawtooth's architecture is that transactions cannot be scheduled on the blockchain itself, as the platform does not have a central clock and would require a consensus algorithm to determine the official system time. This means that scheduled transactions always have to be invoked specifically by a peer. Regular tasks and functions regarding items with an expiration date or a due date (like ADs) have to be scheduled client-side.

This can also cause issues with transaction handler functions whose outputs depend on the current time. Transactions that are submitted close to the cutoff time may be declined because of determinism failures, as system times on different nodes can vary.

On-chain hooks or signals would be a very useful feature for transactions that are triggered by other events. For instance, whenever a new flight is added to the flight log, the aircraft's AMP status should be recalculated, given that many intervals are measured in flight hours or flight cycles. It is possible to listen to these events on individual nodes and then invoke the required function, but this can cause the same transaction to be sent by multiple stakeholders. Transactions that check and update the status of a data model are particularly prone to this (for example AMP tasks that depend on the number of flight cycles/hours). Thus, the transaction would be executed more than once. While this is not a problem in terms of data integrity or security, it does use resources and will block the involved addresses from being written to while the TP is executing the transaction. An on-chain event listener for the AMP model would be a more efficient solution, but this is not possible as of today.

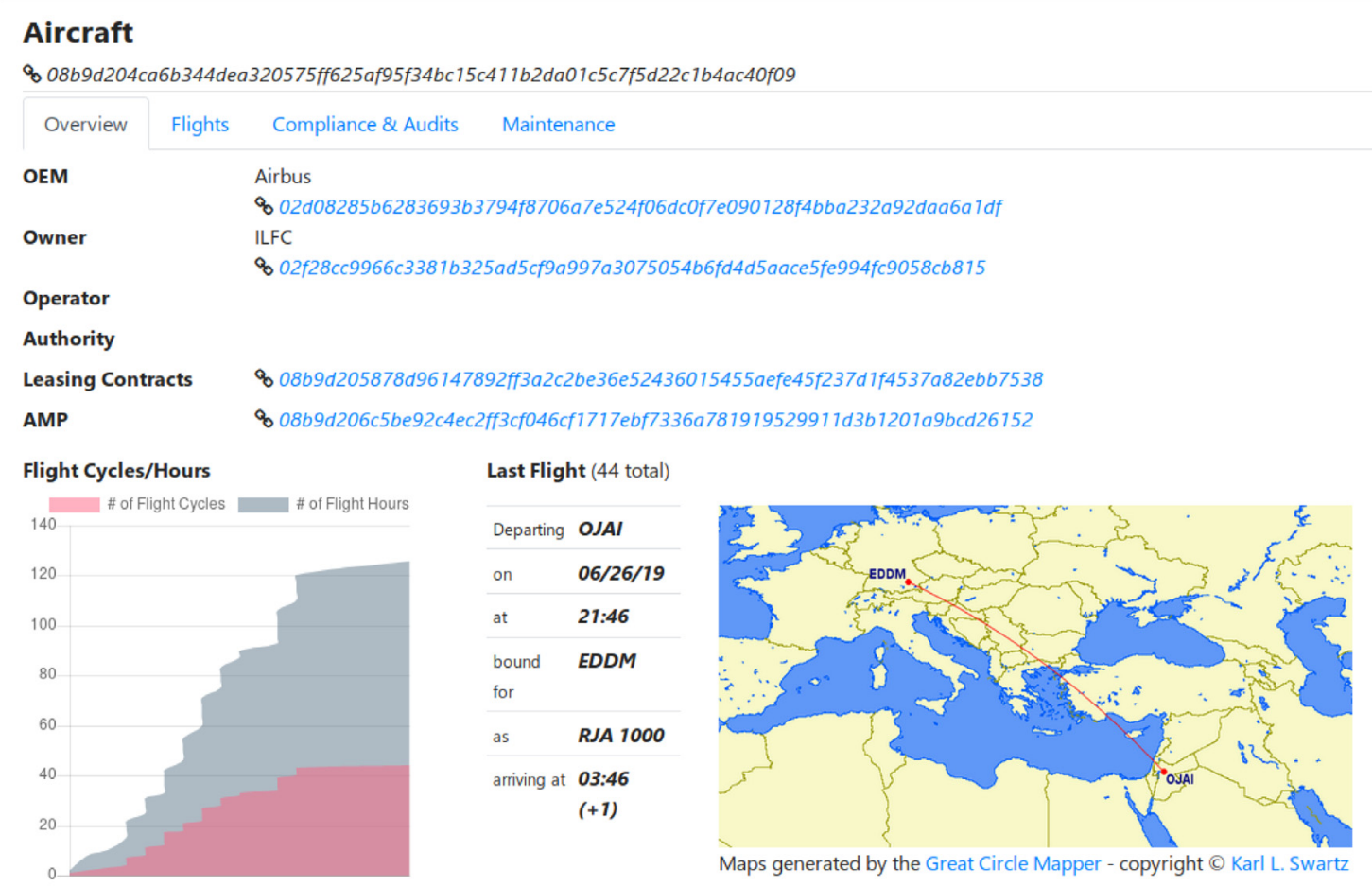

Fig. 12. The Django client showing the aircraft overview page. 
Given that the client converts blockchain data to a relational database schema, the application fulfils C-1. However, the client synchronization appears to be a bottleneck, as the network can process transactions faster than the client's event listener and database can process them. In a production setup, the event listener's traditional ETL (Extract, Transform, and Load) process should be replaced by a streaming ETL process. Possible architectures for this type of data ingestion are discussed in Meehan et al. (2017).

The GUI shows that it is possible to present a complex blockchain-based system like a web 2.0 application, as required by E-5. Further improvements could be made by exposing the node's interfaces through a JavaScript API similar to Ethereum's web3.js. This way, a user could send transactions like audits and file access requests directly from the GUI without having both the request and the response go through the client's backend API.

The compliance requirements in Table 3 could also be validated in the simulation. The blockchain's data models securely store the documentation listed in B-3. Because the aircraft's data model does not change, new stakeholders automatically have access to all relevant data (B-2). With a fully decentralized file storage system, the requirement to store backups in different geographic locations (B-1) will be fulfilled automatically. The current design still requires the stakeholder to implement a compliant backup system. Moreover, data transferability will be automatic, whereas this proof-of-concept uses a TP function that transfers the data to the new stakeholder's server.

As part of the simulation, we measured the blockchain's performance following the guidelines established by The Hyperledger Project (The Hyperledger Project, 2018). A total of 10 nodes were simulated in virtual environments running on a server with the following specifications:

- CPU: Intel ${ }^{\circledR} \mathrm{Xeon}^{\circledR}$ E5-2620 v4

- RAM: $128 \mathrm{~GB}$

- Storage: 128TB SAS3-SSD

The blockchain reached a throughput of 1.68 transactions per second with a maximum latency of 38.67 seconds. It should be noted that the main objective of the simulation was to validate the functionalities of the TPs. While the data access patterns are realistic, the network setup of a real-world implementation (e.g. geographic distribution of nodes) has to be taken into account when simulating a production environment. Also, the simulation was capped at 50 aircraft, but sped up to simulate a full leasing cycle. Therefore, the simulation cannot be viewed as a complete performance test. However, the latency spikes observed in the simulation indicate that other publications that have reported throughputs of 300 (Pongnumkul et al., 2017) or even 700 transactions per second (Dang et al., 2019) seem to arrive at these numbers at least in part because their transaction handlers contain very little or simple business logic.

The maximum latency observed in the simulation is acceptable (C-3). Further work is needed to accurately measure the maximum throughput and the hardware setup necessary to achieve it (the throughput is limited by the slowest node and its CPU in particular (Pongnumkul et al., 2017)). Along the same lines, an estimation of the energy and environmental footprint of the blockchain compared to the traditional paper-based workflow could provide incentives to adopt the platform.

Additional incentives could include a modular standard library with transaction family templates and interfaces to industrystandard software. The solution could also be expanded to include more stakeholders like insurance providers, Air Traffic Control and financial institutions (or decentralized payment processors) natively instead of relying on them as external data sources.
Certain external data providers that have no stake in the blockchain itself could potentially be added to the blockchain as self-updating data models. This could reduce transaction processing times, particularly for those transaction types where network latency is the biggest bottleneck or the external data source is slower than the blockchain itself. While this strategy does not work for very dynamic data like live updates from Air Traffic Control, we have identified some candidates: Aeronautical information is updated in 28 day intervals (AIRAC cycles as defined in International Civil Aviation Organization (2013) Chapter 6) and all changes are announced at least 42 days in advance. This means that the majority of information needed to calculate flight metrics (airport codes, waypoints, etc.) could be stored on the blockchain.

This problem could be used to study the performance implications of external data sources. Particularly valuable conclusions could be drawn regarding the circumstances under which it makes sense to move data to the blockchain and how they can be parametrized (e.g. network latency, time and space complexity of the queries, etc.).

Lastly, while Hyperledger Sawtooth brings a lot of flexibility out of the box, it would be helpful to analyze the performance benefit of compiled and/or statically typed languages like Go in the TP.

\section{Conclusions}

Blockchain offers a set of functionality that can lead to disruptive changes in many industrial sectors, as it is the case with commercial aircraft leasing. This paper analyzes in detail the shortcomings of operational processes and methods in such an industry. Based upon this analysis, it is conducted a mapping between the needs in commercial aircraft leasing and blockchain capabilities. Along the paper, it is shown how automation and workload reduction can be bolstered with the help and guidance of an adequate blockchain arquitecture. For completeness, this roadmap is applied to the design and implementation of a decentralized solution for aircraft leasing.

We have arrived at a proof of concept, but further work is necessary to fully leverage the opportunities of decentralization in each area of this problem. First of all, it is necessary to further explore the convenience of adopting decentralized storage systems for assets management. This analysis should take into account that a centralized approach to file storage may be a necessary intermediate step on the way to a fully decentralized solution. This is due to the fact that decentralized data storage, especially across multiple jurisdictions, still suffers from a lack of legal frameworks and reference cases (Portal, 2020). For instance, an analysis of the legal situation in Germany (Hein et al., 2018) highlights aspects of contract law, liability issues, data protection and criminal law as some of the challenges. It is therefore helpful to show that this concept can be implemented even without decentralized document storage.

The addition of an identity and reputation management system compliant with self-sovereign identity (SSI) principles would further increase traceability and transparency in a way that respects individual liberties and privacy rights. Blockchain-based SSI implementations are discussed in (Ferdous et al., 2019; Baars, 2016), and driven at European level by the European Blockchain Services infrastructure (EBSI). In this context, our solution could also be integrated into a public blockchain platform such as for SSI, notarization, certifications and other legal matters, especially in a business-to-government context such as civil aviation regulatory oversight (Portal, 2020).

While not considered in current civil aviation legislation, machine-to-machine interactions are a central pillar of IoT systems and can potentially be beneficial in aircraft lifecycle management. Many processes that involve multiple parties like flight logging and 
payments are still initiated by human stakeholders, but a decentralized environment would enable the involved machines to carry out these tasks without human input.

This paper demonstrates that the aircraft leasing and lifecycle management sector stands to benefit from a decentralized approach to asset management, which can realistically be handled by a blockchain-based solution. It is clear that a decentralized aircraft leasing system integrates well with other decentralized applications (identity management, payments, supply chains, insurance, etc.) and will deliver more value in a more developed ecosystem. More and more companies in the aviation industry have included decentralized solutions in their IoT strategy, and it is only a matter of time until the first blockchain-based aircraft lease agreements are going to appear.

\section{Authors' contributions}

Paul Kuhle: Conceptualization, Methodology, Investigation, Software, Visualization, Writing - Original Draft, Writing - Review \& Editing.

David Arroyo: Conceptualization, Methodology, Investigation, Writing - Original Draft, Writing - Review \& Editing.
Eric Schuster: Conceptualization, Writing - Original Draft, Writing - Review \& Editing. All authors have read and agreed to the published version of the manuscript.

\section{Declaration of Competing Interest}

The authors report no declarations of interest.

\section{Acknowledgements}

This work has been supported by the Comunidad de Madrid (Spain)under the project CYNAMON (P2018/TCS-4566), cofinanced with FSE andFEDER EU funds, by Ministerio de Economía, Industria y Competitividad (MINECO), Agencia Estatal de Investigación (AEI), and European Regional Development Fund (ERDF, EU), through project COPCIS, grant number TIN2017-84844-C2-1$\mathrm{R}$, and by the Consejo Superior de Investigaciones Científicas (CSIC) under the project LINKA20216 ("Advancing in cybersecurity technologies", i-LINK+ program). We thank flightera.net for providing us with flight data for the simulation.

\section{Appendix A. Different blockchain types}

Table 2

A comparison of different blockchain types. Adapted and modified from (Tamayo, 2016).

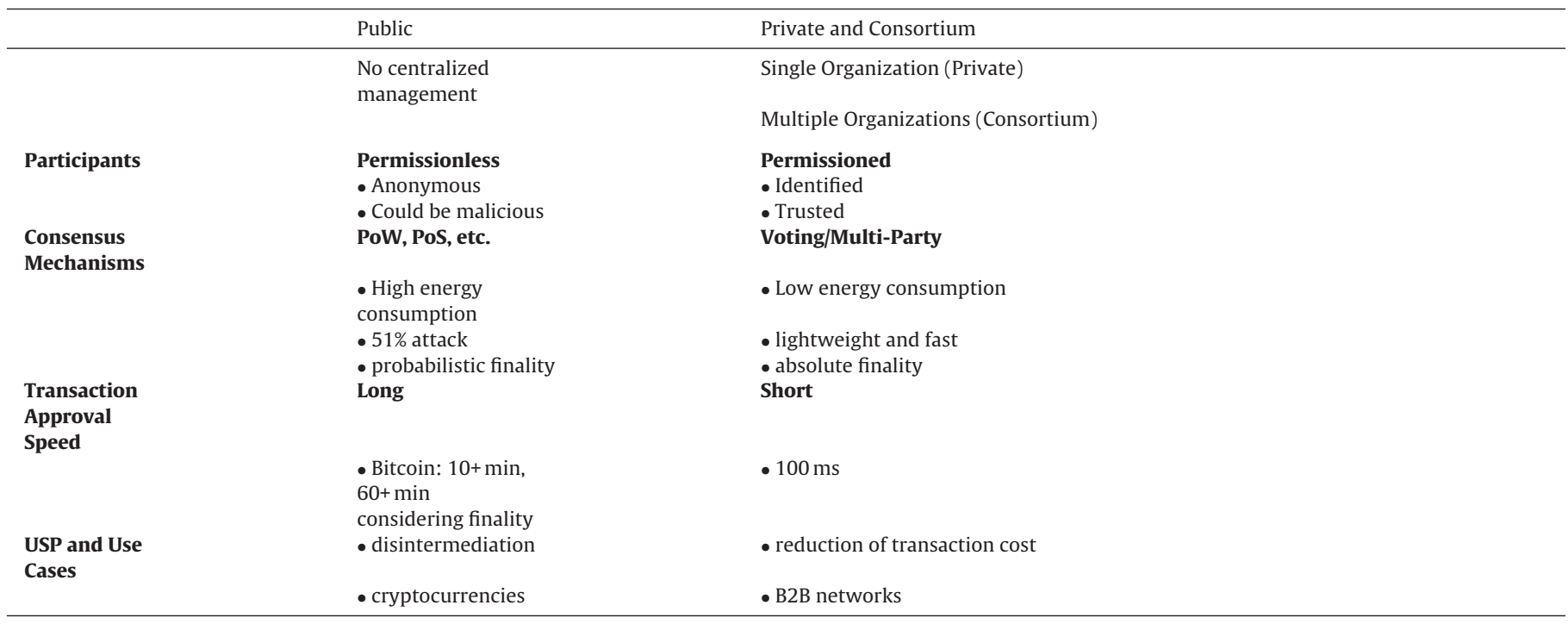




\section{Appendix B. Non-functional requirements}

Table 3

Non-functional requirements: compliance.

\begin{tabular}{|c|c|c|}
\hline $\mathrm{Nr}$ & Title & Description \\
\hline B-1 & Data Protection & $\begin{array}{l}\text { All records have to be stored "in a manner that ensures protection from damage, alteration and theft" (M.A.714). } \\
\text { Digital backups have to be stored in a different location. }\end{array}$ \\
\hline B-2 & $\begin{array}{l}\text { Data } \\
\text { Transferability }\end{array}$ & $\begin{array}{l}\text { The CAMO has to ensure that all records can be transferred in case the CA activity is transferred to a different } \\
\text { organization. }\end{array}$ \\
\hline B-3 & Documentation & $\begin{array}{l}\text { The CAMO is required to keep aircraft and engine logbooks with flight records, CA/MX records as well as the } \\
\text { aircraft's build stand and equipment list, as described in M.A.305. Paragraph M.A.714 requires the CAMO to retain } \\
\text { copies of these records "until two years after the aircraft has been permanently withdrawn from service". }\end{array}$ \\
\hline
\end{tabular}

All requirements in this table are based on Commission Regulation (EU) No 1321/2014, Annex I (EU, 2014), specifically articles M.A.305, M.A.709 and M.A.714.

Table 4

Non-functional requirements: performance.

\begin{tabular}{|c|c|c|}
\hline $\mathrm{Nr}$ & Title & Description \\
\hline$C-1$ & Scalability & $\begin{array}{l}\text { With about } 25,000 \text { commercial aircraft in service as of } 2017 \text { and a forecast of 35,000 aircraft in 2027, the } \\
\text { scalability of the platform is crucial (Cooper et al., 2017). While the performance of a blockchain is typically } \\
\text { measured in transaction throughput and transaction latency, data storage and on-chain accounting for audits are } \\
\text { also relevant metrics in the context of this particular application. Data storage costs should be minimized, and } \\
\text { queries should not perform significantly worse than in a system with relational databases. }\end{array}$ \\
\hline $\mathrm{C}-2$ & Throughput & $\begin{array}{l}\text { With a conservative estimate of } 20 \text { daily write requests per aircraft (flight logs, MX events, etc.) and 35,000 } \\
\text { aircraft in service, the blockchain would need to be able to process 700,000 transactions per day (about } 8 \\
\text { transactions per second). }\end{array}$ \\
\hline$C-3$ & Latency & $\begin{array}{l}\text { As opposed to many blockchain applications in the financial sector, commercial aircraft leasing is not a } \\
\text { low-latency application. However, transactions should be finalized within a reasonable timeframe }(\sim 10-30 \mathrm{~min}) \\
\text { to allow for same-day audits. }\end{array}$ \\
\hline
\end{tabular}

Table 5

Non-functional requirements: business needs.

\begin{tabular}{|c|c|c|}
\hline $\mathrm{Nr}$ & Title & Description \\
\hline D-1 & $\begin{array}{l}\text { Data } \\
\text { Ownership }\end{array}$ & $\begin{array}{l}\text { Given the sensitive nature of the data stored on this blockchain, the individual stakeholders will want to retain } \\
\text { ownership of their data. This means that files will either have to be stored on-chain or on a server of the data } \\
\text { owner's choosing. Both solutions require an access control system managed by the blockchain. }\end{array}$ \\
\hline D-2 & $\begin{array}{l}\text { Operating } \\
\text { Costs }\end{array}$ & $\begin{array}{l}\text { While enterprise blockchains are often implemented to increase efficiency and reduce costs (Al-Jaroodi and } \\
\text { Mohamed, 2019), it is important to understand the costs associated with operating a large-scale blockchain. } \\
\text { Particularly, the energy footprint of blockchains has been the subject of debate (Vranken, 2017) and should be } \\
\text { taken into consideration. }\end{array}$ \\
\hline
\end{tabular}

Table 6

Non-functional requirements: usability.

\begin{tabular}{|c|c|c|}
\hline $\mathrm{Nr}$ & Title & Description \\
\hline E-1 & Integration & $\begin{array}{l}\text { The platform will be integrated into a landscape of legacy IT solutions (e.g. MRO software). Therefore, the solution } \\
\text { needs to integrate well into legacy systems, but emphasize and support an increasingly decentralized architecture } \\
\text { (e.g. decentralized payments and supply chain tracking). }\end{array}$ \\
\hline E-2 & Flexibility & $\begin{array}{l}\text { Given the different business needs of the involved stakeholders, each party may wish to customize their } \\
\text { blockchain node (e.g. for data synchronization, interfaces to other systems). The architecture of the blockchain } \\
\text { platform should avoid vendor lock-in, specifically with regards to hosting services and programming languages. }\end{array}$ \\
\hline E-3 & Ecosystem & $\begin{array}{l}\text { Smaller stakeholders may not have the resources to maintain their own nodes or to develop custom software to } \\
\text { integrate them into their existing IT infrastructure. Therefore, the solution should facilitate the development of an } \\
\text { ecosystem around the blockchain platform. This ecosystem would enable third parties to offer solutions like } \\
\text { managed hosting and custom blockchain clients. }\end{array}$ \\
\hline E-4 & Maintainability & $\begin{array}{l}\text { Given the long time horizon of this project, a focus on maintainability and support is essential. The underlying } \\
\text { blockchain technology and the libraries used for core functionalities should be mature and offer long-term } \\
\text { enterprise support. }\end{array}$ \\
\hline E-5 & $\begin{array}{l}\text { Interface } \\
\text { Design }\end{array}$ & $\begin{array}{l}\text { The end user should not need blockchain-specific knowledge or training to be able to use the platform. GUIs for } \\
\text { humans should behave like web } 2.0 \text { applications. Other systems should be able to communicate with the } \\
\text { blockchain system using established industry standards like REST APIs. }\end{array}$ \\
\hline
\end{tabular}




\section{Appendix C. Data models}

Listing 1.1: Data model for stakeholders

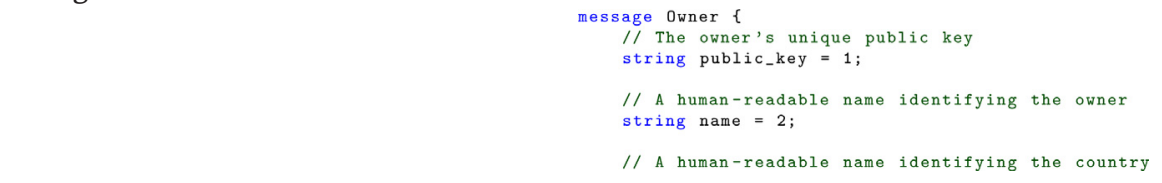

Listing 1.2: Data model for aircraft

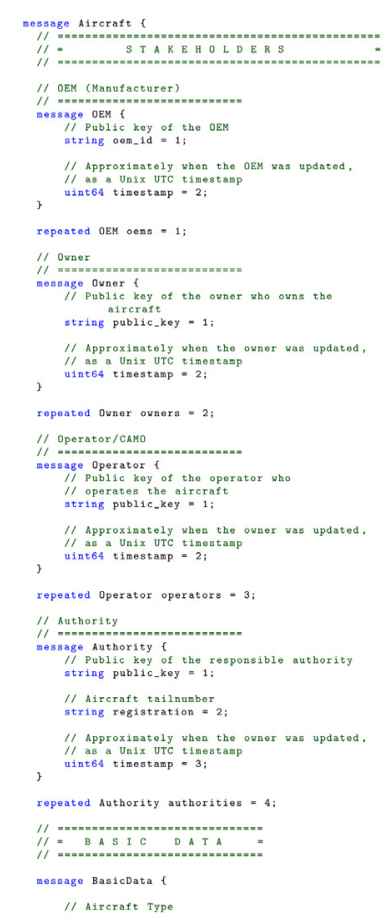

Listing 1.3: Data model for AMPs
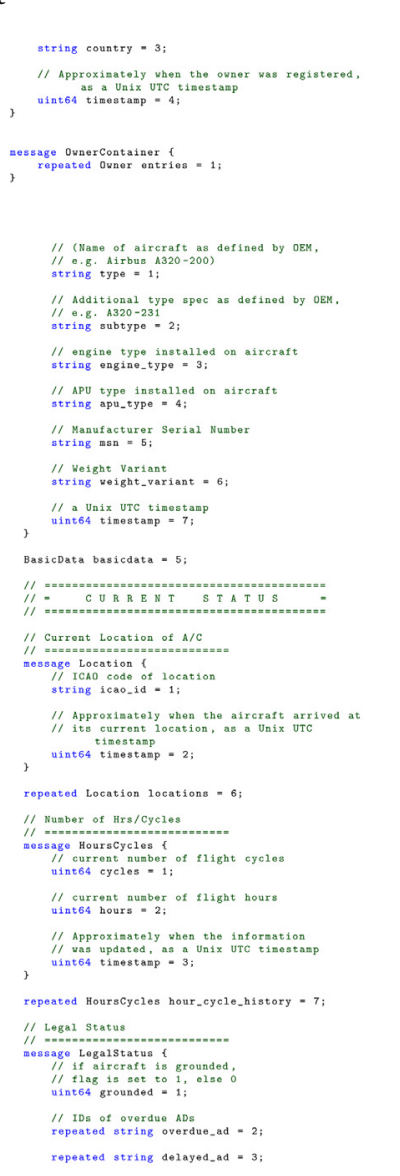

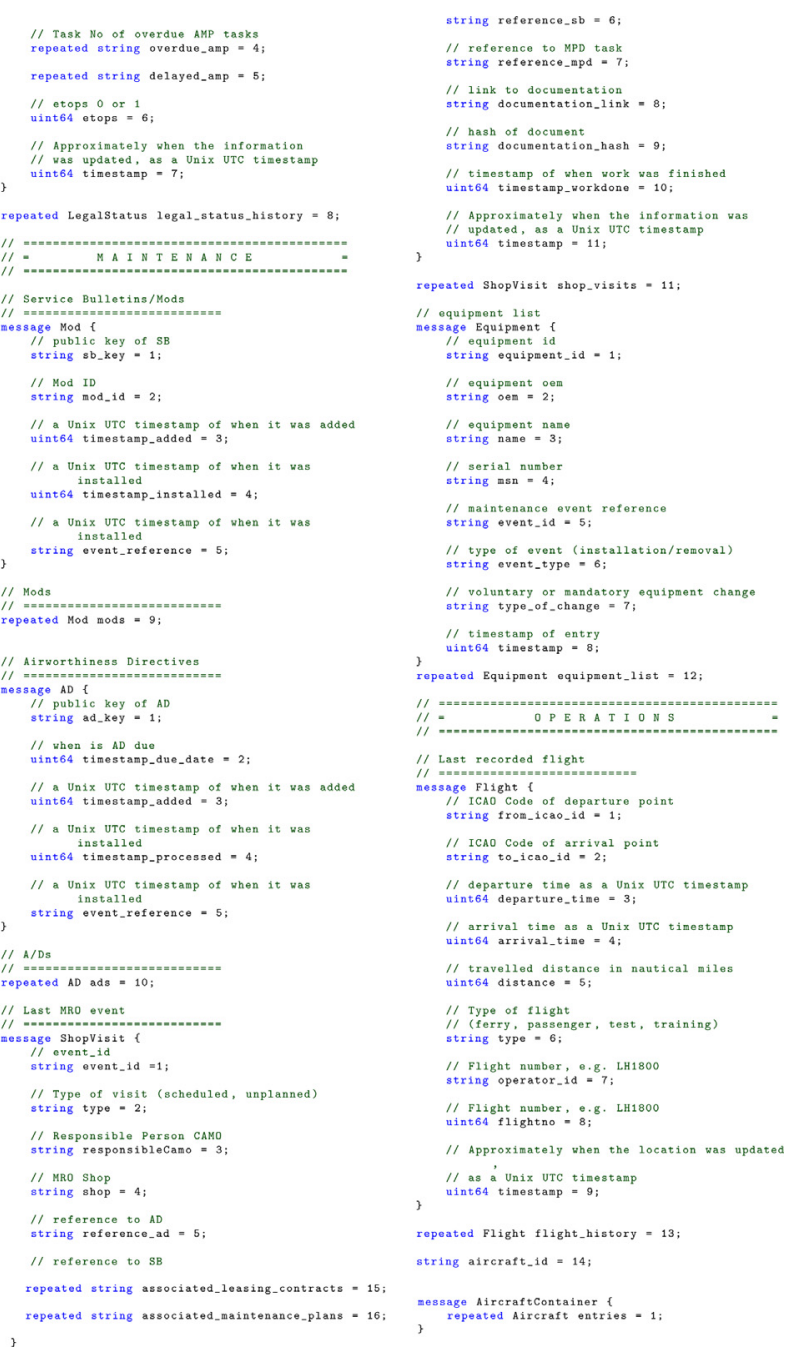




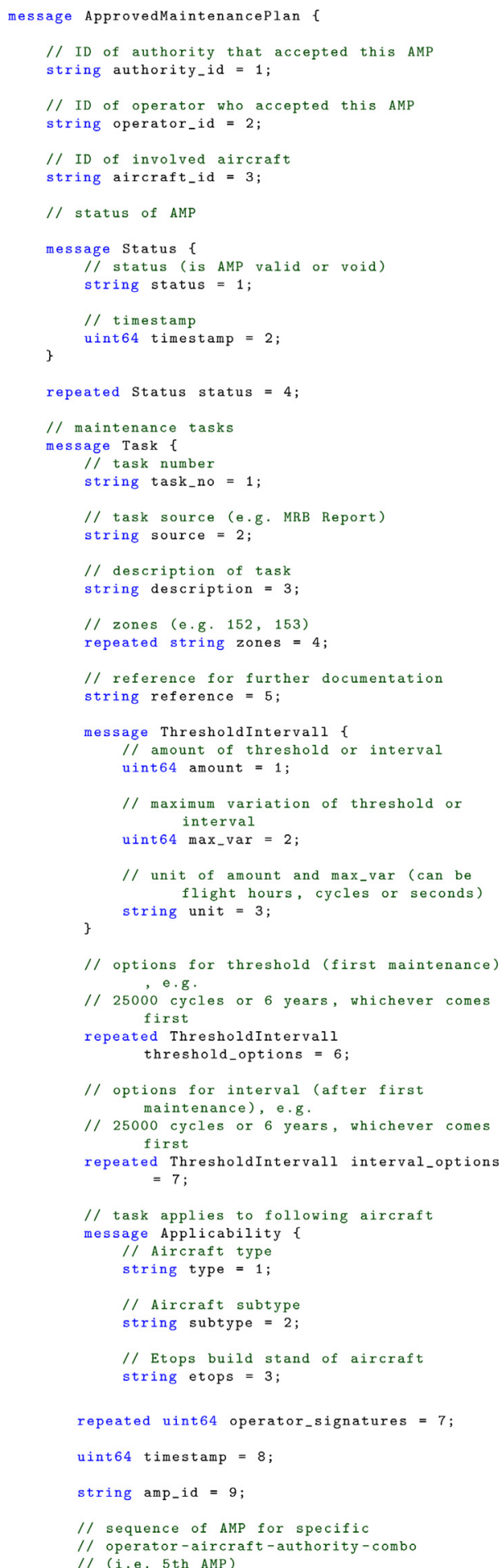

Listing 1.4: Data model for leasing contracts

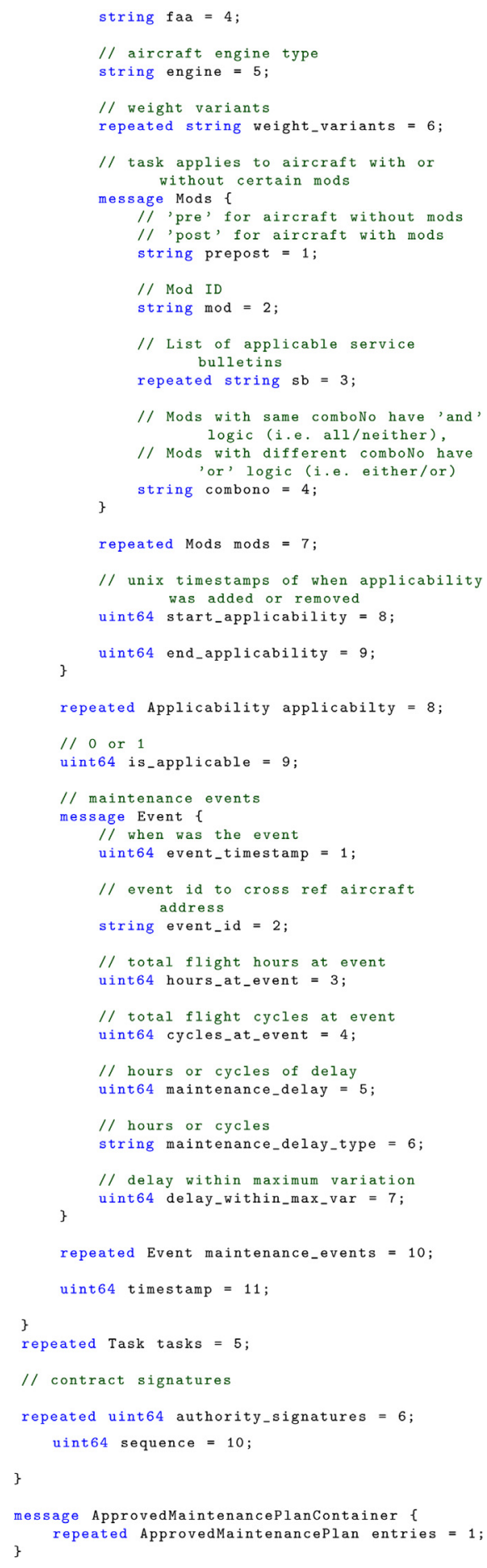




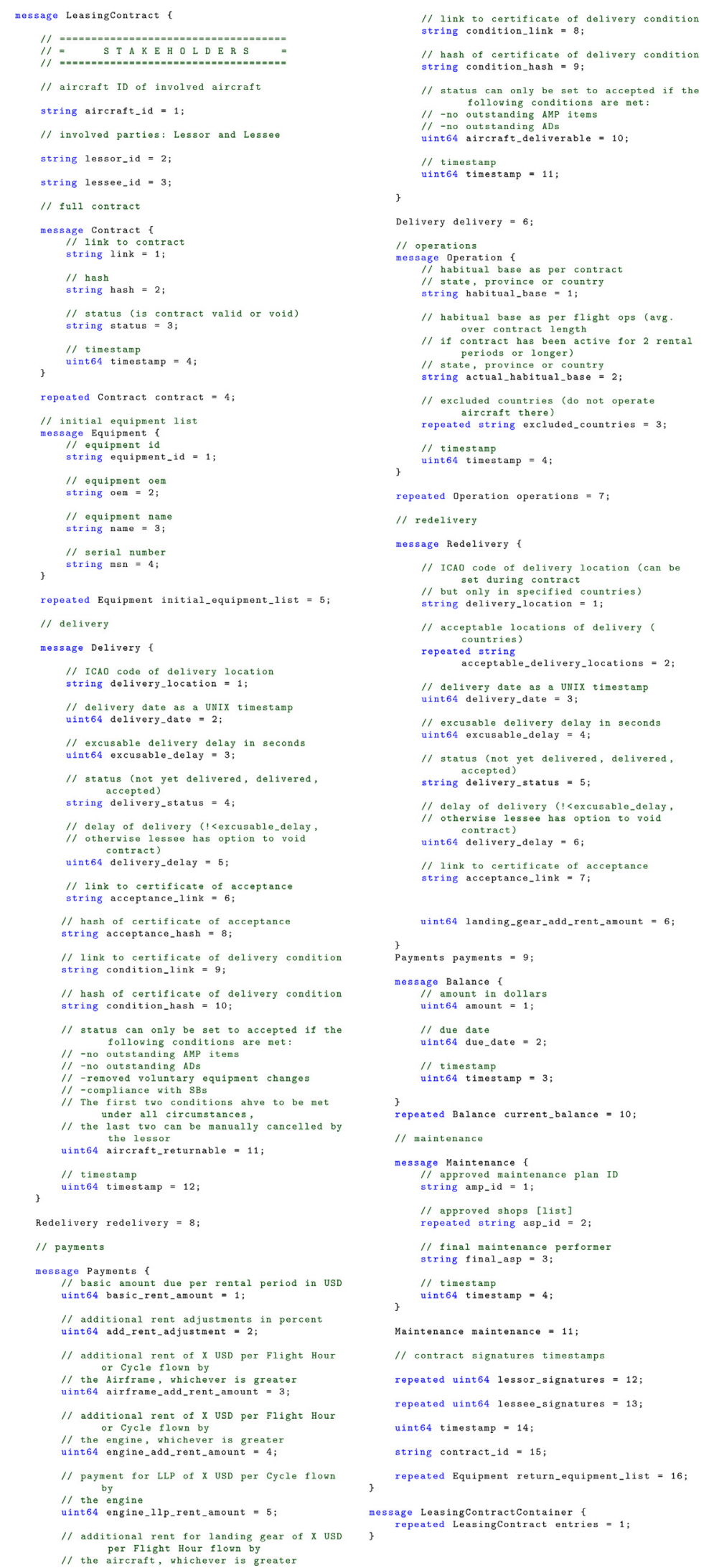

Listing 1.5: Data model for transaction payloads

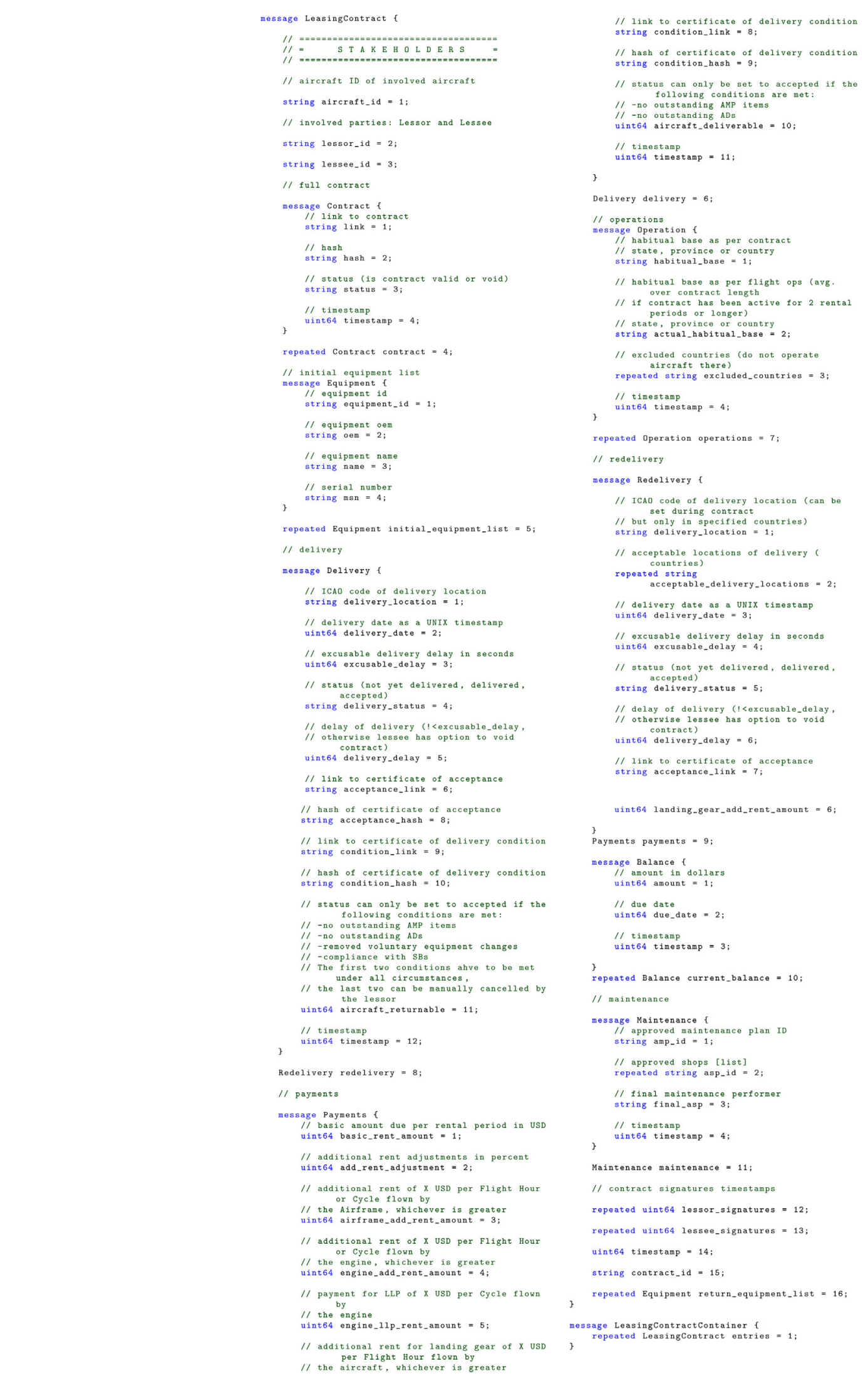




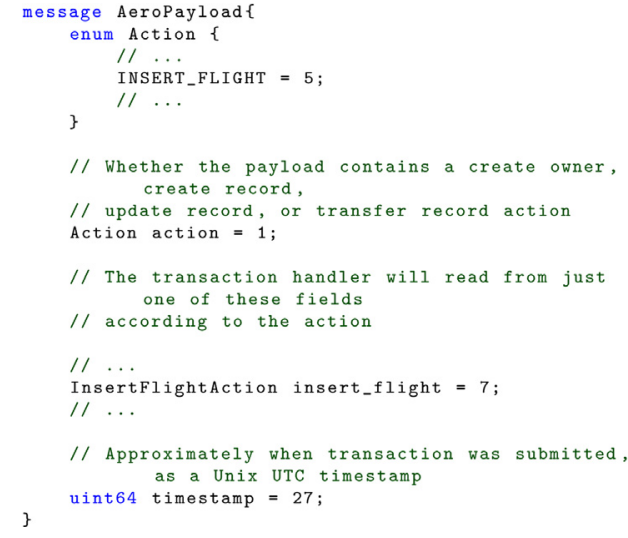

// departure airfield string dep_icao $=2$;

$/ /$ arrival airfield

string arr $-i c a o=3$;

// time of departure

string timestamp_dep $=4$

$/ /$ time of arrival

string timestamp_arr $=5$

// Type of flight

string type $=6$;

// operator_id

string operator_id $=7$

// flight number

uint 64 flightno $=8$;

Appendix D. Django application

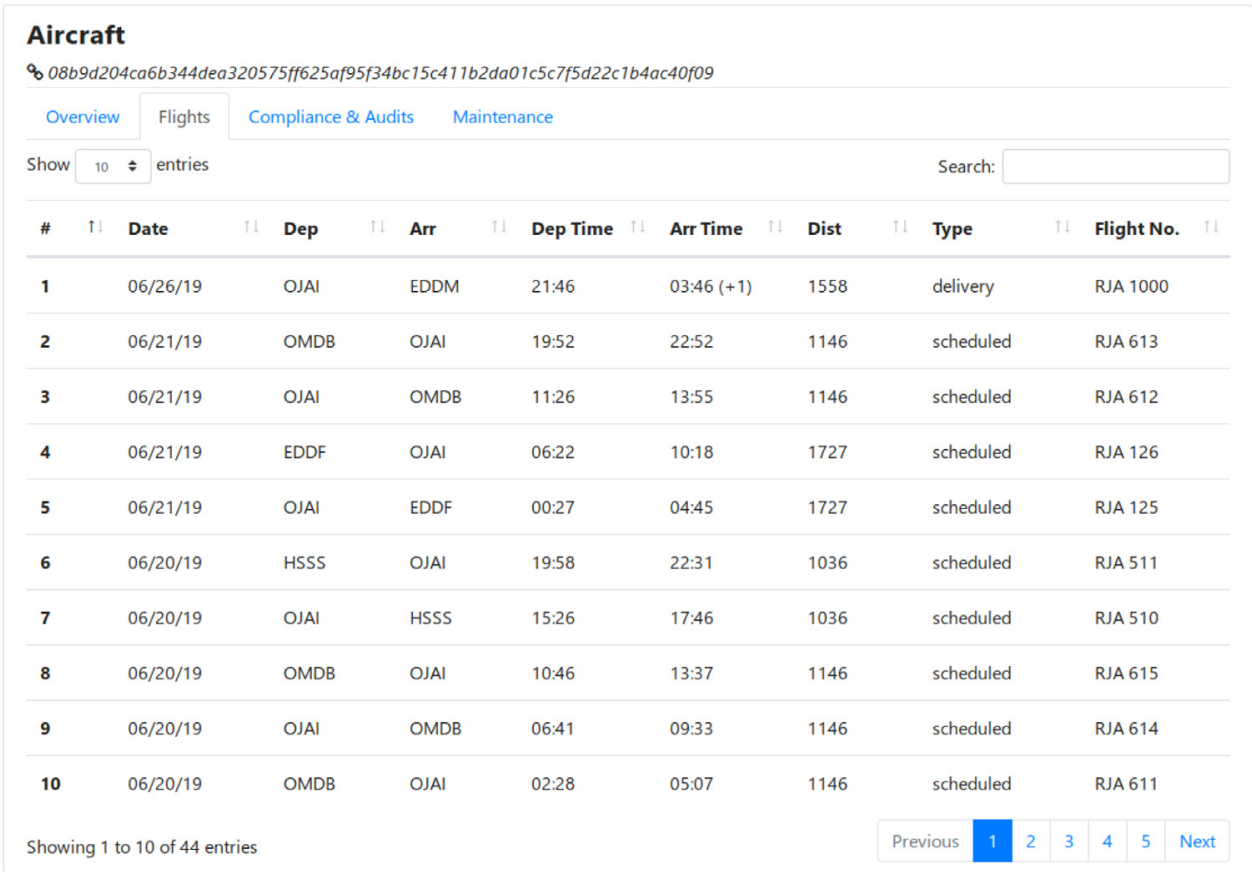

Fig. 13. The Django client showing the aircraft's flight logs. 


\section{Approved Maintenance Plan}

B 08b9d206c5be92c4ec2ff3cf046cf1717ebf7336a781919529911d3b1201a9bcd26152

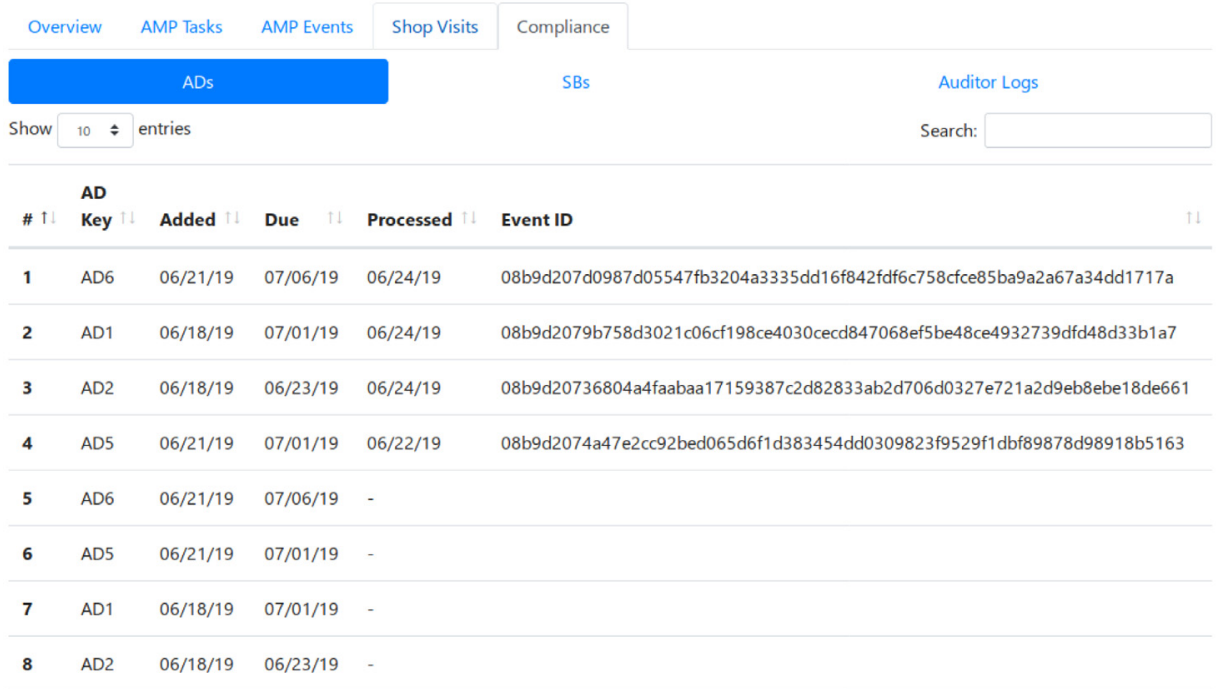

Fig. 14. The Django client showing the aircraft's AMP page.

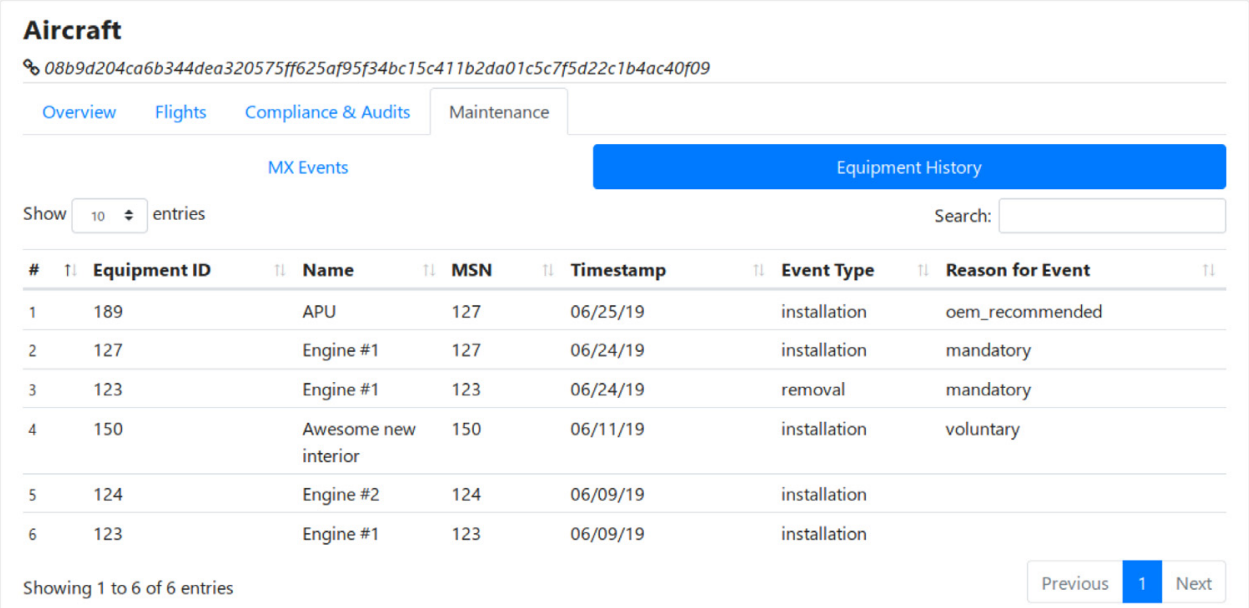

Fig. 15. The Django client showing the aircraft's equipment history page. 


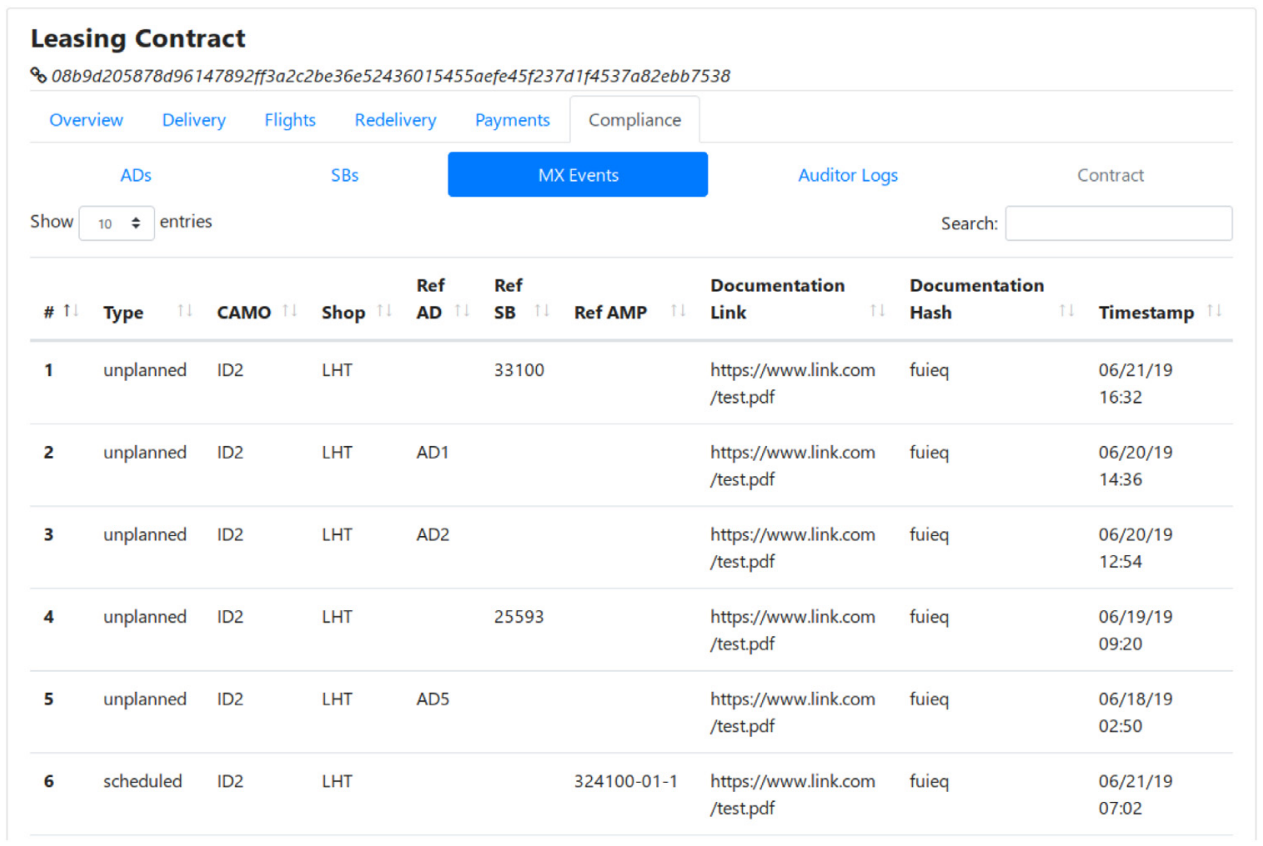

Fig. 16. The Django client showing the aircraft's MX history.

\section{Leasing Contract}

Bo 08b9d205878d96147892ff3a2c2be36e52436015455aefe45f237d1f4537a82ebb7538

\begin{tabular}{|c|c|c|c|c|c|c|c|c|}
\hline Overview & Delivery & Redelivery & Payments & liance & & & & \\
\hline \multirow[t]{7}{*}{ Payments } & & Basic Rent per Rental Period & $\$ 15,000$ & Billing & $\#$ & Invoice Date & Due Date & Amount \\
\hline & & Basic Rent Adjustment per Year & $1 \%$ & & 1 & $06 / 25 / 19$ & $07 / 25 / 19$ & $\$ 37,564$ \\
\hline & & Add Rent for Airframe per FH & $\$ 150$ & & & & & \\
\hline & & Add Rent for Engines per FC & $\$ 75$ & & & & & \\
\hline & & Add Rent for Engine LLP per FH & $\$ 75$ & & & & & \\
\hline & & Add Rent for Landing Gear per FH & $\$ 90$ & & & & & \\
\hline & & Add Rent will be collected together & with Basic Rent. & & & & & \\
\hline
\end{tabular}

Fig. 17. The Django client showing a leasing contract's payment page. 


\section{References}

Al-Jaroodi, J., Mohamed, N., 2019]. Blockchain in industries: a survey. IEEE Access 7, 36500-36515

Arribas, I., Arroyo, D., Reshef Kera, D., 2020]. Sandbox for minimal viable governance of blockchain services and daos: Claudia. In: Prieto, J., Pinto, A., Das, A.K., Ferretti, S. (Eds.), Blockchain and Applications. Springer International Publishing, Cham, pp. 24-30.

2020]. Aviation Working Group: Global Aircraft Trading System. http://www.awg. aero/project/gats/.

Baars, D., 2016]. Towards Self-Sovereign Identity Using Blockchain Technology Master's Thesis. University of Twente.

Cooper, T., Smiley, J., Porter, C., Precourt, C., 2017]. Global Fleet \& MRO Market Forecast Summary 2017-2027 (June)

Dang, H., Dinh, T.T.A., Loghin, D., Chang, E.C., Lin, Q., Ooi, B.C., 2019]. Towards scaling blockchain systems via sharding. In: Proceedings of the 2019 International Conference on Management of Data. SIGMOD'19. ACM, New York, NY, USA, pp. 123-140, http://dx.doi.org/10.1145/3299869.3319889.

Di Vaio, A., Varriale, L., 2020]. Blockchain technology in supply chain management for sustainable performance: evidence from the airport industry. Int. J. Inf. Manag. 52, 102014.

ouncil of the European Union: Regulation (european parliament and of the council of 20 february 2008 on common rules in the field of civil aviation and establishing a european aviation safety agency, and repealing council directive 91/670/eec, regulation (ec) no 1592/2002 and directive 2004/36/ec., 2008. Off. J. Eur. Union L 79/1 (March).

ouncil of the European Union: Commission regulation (. Official Journal of the European Union L 79/1 (March 2008). Council of the European Union: Commission regulation (eu) no, 1321Council of the European Union: Commission regulation (eu) no 1321/2014 of 26 november 2014 on the continuing airworthiness of aircraft and aeronautical products, parts and appliances, and on the approval of organisations and personnel involved in these tasks., 2014. Off. J. Eur. Union L 362/1 (December).

ouncil of the European Union: Regulation (e. Official Journal of the European Union L 362/1 (December 2014).Council of the European Union: Regulation (eu), 2018Council of the European Union: Regulation (eu) 2018/1139 of the european parliament and of the council of 4 european union aviation safety agency, and amending regulations (ec) no 2111/2005, (ec) no 1008/2008, (eu) no 996/2010, (eu) no $376 / 2014$ and directives 2014/30/eu and 2014/53/eu of the european parliament and of the council, and repealing regulations (ec) no 552/2004 and (ec) no 216/2008 of the european parliament and of the council and council regulation (eec) no 3922/91., 2018. c) no, 20080ff. J. Eur. Union L 212/1 (August).

2019]. Federal Aviation Administration: FAA Proposes $\$ 715,438$ Civil Penalty Against Allegiant Air. https://www.faa.gov/news/press_releases/news_story. cfm?newsId $=23798$.

Ferdous, M.S., Chowdhury, F., Alassafi, M.O., 2019. n search of self-sovereign identity leveraging blockchain technology. IEEE Access 7, 103059-103079.

2019]. Forum, W.E.: Inclusive Deployment of Blockchain for Supply Chains: Part 1 Introduction, Tech. Rep. http://www3.weforum.org/docs/WEF_Introduction_to_ Blockchain_for_Supply_Chains.pdf.

2019]. Google LLC: Protocol Buffers. https://developers.google.com/protocolbuffers/.

Hanley, D.P., 2011]. Aircraft Operating Leasing: A Legal and Practical Analysis in the Context of Public and Private International Air Law. Dissertation, Leiden University https://openaccess.leidenuniv.nl/handle/1887/18146.

Hein, C., Wellbrock, W., Hein, C., 2018]. Rechtliche Herausforderungen von Blockchain-Anwendungen: Straf-, Datenschutz-und Zivilrecht. Springer.

Heiss, J., Eberhardt, J., Tai, S., 2019]. From oracles to trustworthy data on-chaining systems. In: 2019 IEEE International Conference on Blockchain (Blockchain), IEEE, pp. 496-503.

Huang, H., Lin, J., Zheng, B., Zheng, Z., Bian, J., 2020. hen blockchain meets distributed file systems: An overview, challenges, and open issues. IEEE Access 8IEEE Access 8, 50574-50586.

2020]. Hyperledger: Supporting Members. https://www.hyperledger.org/about/ members.

2012]. International Air Transport Association, A: Master Short-Term Engine Lease Agreement. http://www.awg.aero/assets/docs/Commentary-STEEL.PDF.
2017]. International Air Transport Association: Guidance Material and Best Practices for Aircraft Leases, 4th ed. International Air Transport Association.

2018]. International Air Transport Association: Blockchain in Aviation. https://www. iata.org/publications/Documents/blockchain-in-aviation-white-paper.pdf.

2001]. International Civil Aviation Organization, I.: The Convention on International Interests in Mobile Equipment, and Its Protocol on Matters Specific to Aircraft Equipment. https://www.unidroit.org/instruments/security-interests/ cape-town-convention.

2013]. International Civil Aviation Organization: Annex 15 to the Convention on International Civil Aviation: Aeronautical Information Services. International Civil Aviation Organization.

2019]. Intel Corporation: Hyperledger Sawtooth Documentation. https://sawtooth. hyperledger.org/docs/core/releases/1.0/contents.html.

Kehoe, L., Hallahan, J., 2017. Blockchain -- a game changer in aircraft leasing? Air Finance Journal10Air Finance J. 1, 84-87 https://www2.deloitte.com/content/ dam/Deloitte/ie/Documents/Tax/ie-blockchain-a-game-changer-in-aircraftleasing.pdf.

Lao, L., Li, Z., Hou, S., Xiao, B., Guo, S., Yang, Y., 2020. survey of iot applications in blockchain systems: Architecture, consensus, and traffic modeling. ACM Computing Surveys (CSUR) 53 (1), 1-32.

Li, J., Li, N., Peng, J., Cui, H., Wu, Z., 2019. nergy consumption of cryptocurrency mining: A study of electricity consumption in mining cryptocurrencies. Energy 168, 160-168 http://www.sciencedirect.com/science/article/pii/ S0360544218322503.

Meehan, J., Aslantas, C., Zdonik, S., Tatbul, N., Du, J., 2017. Data ingestion for the connected world. CIDR.

Nadimi, F., 2019]. How Sanctions are Affecting Iran's Airline Industry. https://www. washingtoninstitute.org/policy-analysis/view/how-sanctions-are-affectingirans-airline-industry.

Narayanan, A., Clark, J., 2017. itcoin's academic pedigree. Commun. ACM6012 (NovCommun. ACM 60 ()), 36-45, http://dx.doi.org/10.1145/3132259.

Pongnumkul, S., Siripanpornchana, C. Thajchayapong, S., 2017]. Performance analysis of private blockchain platforms in varying workloads. 2017 26th International Conference on Computer Communication and Networks (ICCCN), 1-6.

Portal, E.D., 2020]. Analytical Report 12: Business-to-Government Data Sharing Tech. Rep. https://www.europeandataportal.eu/sites/default/files/analytical report_12_business_government_data_sharing.pdf.

Radin, A., Reach, C., 2017]. Russian Views of the International Order, 1st ed. RAND Corporation.

Rubio, J.E., Alcaraz, C., Rios, R., Roman, R., Lopez, J., 2020]. Distributed detection of apts: consensus vs. clustering. European Symposium on Research in Computer Security, 174-192.

2019]. S7 Airlines: The Amount of Operations via the s7 Airlines Blockchain Platform and Alfa-Bank Exceeded \$1 Million in July. https://www.s7.ru/en/about/news/ the-amount-of-operations-via-the-s7-airlines-blockchain-platform-and-alfabank-exceeded-1-million-in-july/.

Tamayo, D., 2016]. Making Blockchain Real for Business: Explained. https://www. slideshare.net/DiegoDiaz49/1-ibm-blockchain-explained.

2017]. The Hyperledger Project: Hyperledger Architecture, Volume 1: Introduction to Hyperledger Business Blockchain Design Philosophy and Consensus. https://www.hyperledger.org/wp-content/uploads/2017/08/HyperledgerArch_WG_Paper_1_Consensus.pdf.

2018]. The Hyperledger Project: Hyperledger Blockchain Performance Metrics. https://www.hyperledger.org/wp-content/uploads/2018/10/HL_Whitepaper_ Metrics_PDF_V1.01.pdf.

2008]. Transport Studies Group: Dynamic Cost Indexing. Innovative Cooperative Actions of R\&D in EUROCONTROL Programme CARE INO III.

Vranken, H., 2017. ustainability of bitcoin and blockchains. Current Opinion in Environmental SustainabilityCurr. Opin. Environ. Sustain. 28, 1-9, sustainability governance http://www.sciencedirect.com/science/article/pii/ S1877343517300015.

Yang, Y., Cooper, D., Collomosse, J., Dragan, C., Manulis, M., Briggs, J., Steane, J., Manohar, A., Moncur, W., Jones, H., 2020. Tapestry: A de-centralized service for trusted interaction online. IEEE Transactions on Services ComputingIEEE Trans. Serv. Comput.

Yermack, D., 2017. orporate governance and blockchains. Review of Finance 21 (1), 7-31. 